\title{
ADDRESSING SEASONAL VARIATIONS TO PREDICT CONSUMER PRICES OF RED MEAT
}

\author{
WAFAA A. M. EID
}

Cent. Lab. For Design \& Stat. Res., ARC, Egypt.

(Manuscript received 5 December 2017)

\begin{abstract}
$\mathrm{T}$ he study aims to calculate the seasonal index of the prices of red meat (Sheep meat (Dani), Buffalo meat (Jamousi) and Cow meat (Kunduz) during the period (2008-2016) to be used as a guide in decision making of production and consumption and developing the agricultural economic policies. The study used the methodology of Box-Jenkins to predict monthly red meat prices. It was found that the optimal model is [ ARIMA $(1.0 .1) \mathrm{S}(1,1,1)]$ for buffalo meat, and the best model for monthly prices model for cow meat (Kunduz) is $[0,1,2] \mathrm{S}(2,0,2)]$ and the best model for forecasting monthly prices of sheep meat (Dani) is (ARIMA) $(0,1,2) \mathrm{S}(1.0,0)]$. Also, the demand of consumers to buy meat is in October and November during the national holidays and that there is convergence between predictive values and actual values. The study recommends using these models to predict the monthly prices of red meat (Jamousi, Kunduz and Dani).
\end{abstract}




\title{
معالجة التغير ات الموسمية للتنبؤ \\ بأسعار المستهلك للحوم الحمراء
}

\author{
وفاء أبو بكر محمد عيد \\ المعمل المركزي لبحوث التصميم والتحليل الإحصائي _مركز البحوث الزراعية

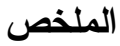

يستهدف البحث حساب الدليل الموسمي لأسعار اللحـوم الحمــر اء (الضـــاني، الجاموســي

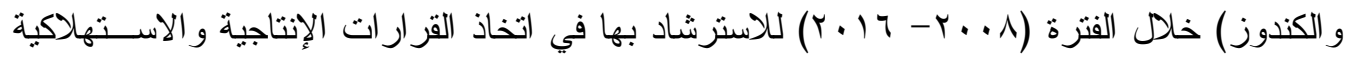
ووضع السياسات الاقتصادية الزر اعية، و استخدم البحث منهجية Box-Jenkins للتنبؤ بأسعار اللحــوم

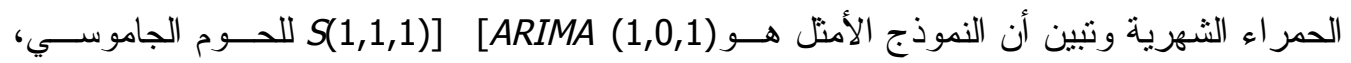

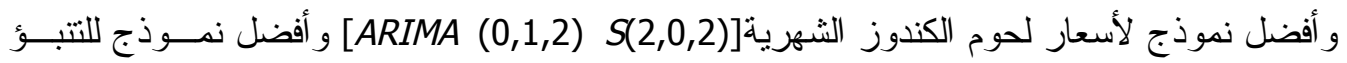
بأسعار اللحوم الضاني الثهري [ARIMA $(0,1,2)$ S(1,0,0)، كما تنين إقبال المستهلكين علي شر اء

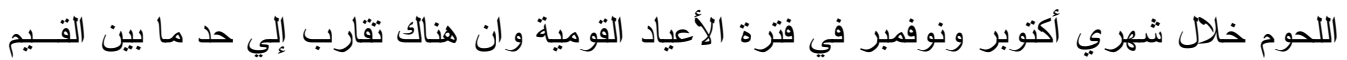

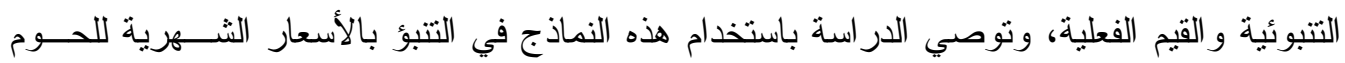
الحمر اء (الضاني، الجاموسي و الكندوز ).

\section{مقدمة}

يعد التتبؤ بالسلوك المستقبلي للسلاسل الزمنية من الموضوعات المهمة في العلوم الإحصائية

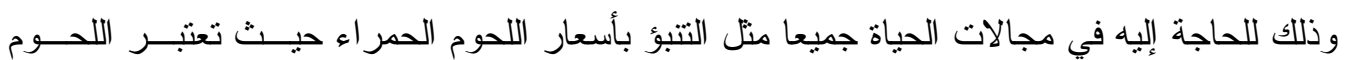
مصدراً هاماً ورئيسياً لتوفير البروتين الحيو اني اللازم لغذاء الإنسان في مصر ، ومع نز ايــــ أعــداد

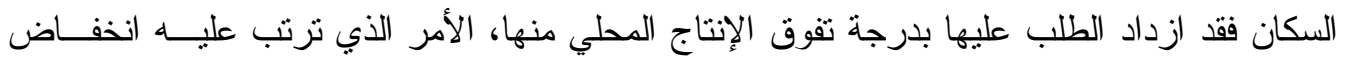

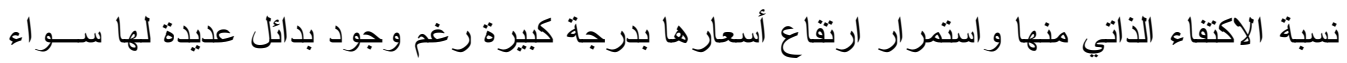

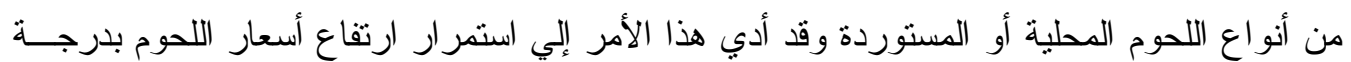
كبيرة لا تتتاسب مع دخول المستهكين في مصر الأمر الذي ترتب عليه انخفاض نصيب الفرد مــن

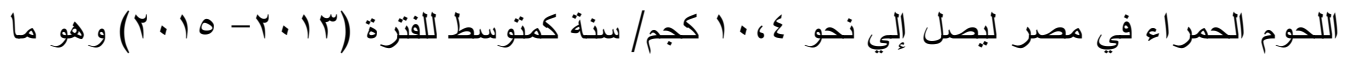

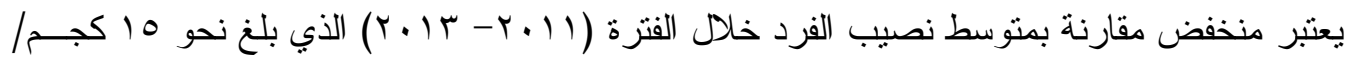

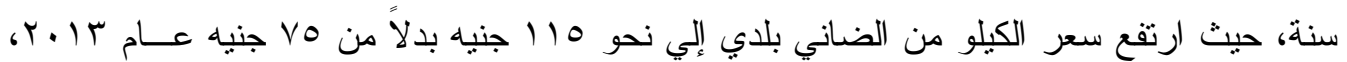

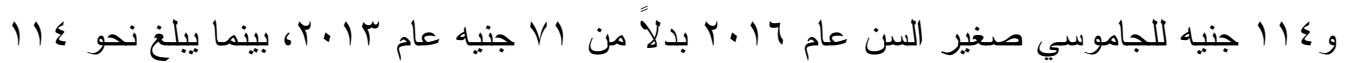

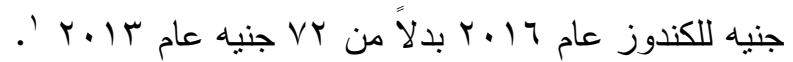




\section{مشكلة البحث}

تفتقر التحليلات الإحصائية للسلاسل الزمنية لأسعار استهلاك اللحوم الحمــر اء إلــي الدقـــة

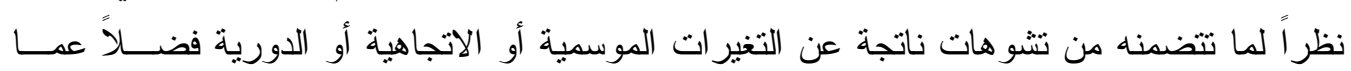

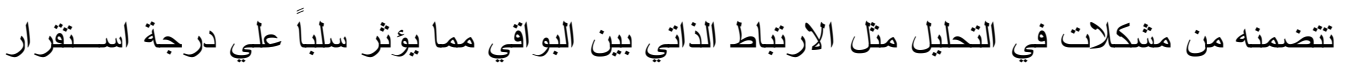

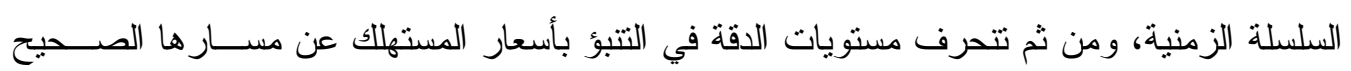

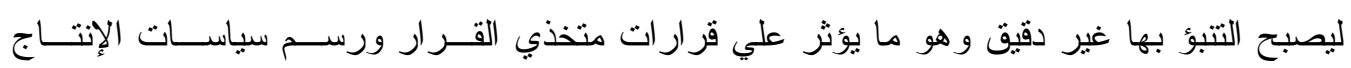

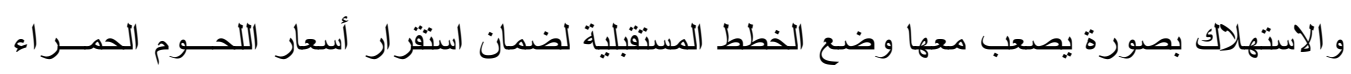
خلال شهور السنة.

\section{هدف البحث}

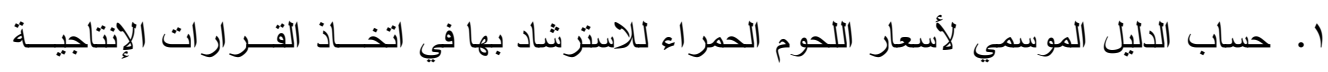

$$
\text { و الاستهلاكية التي قد تساعد و اضعي السياسات الاقتصادية الزر اعية. }
$$

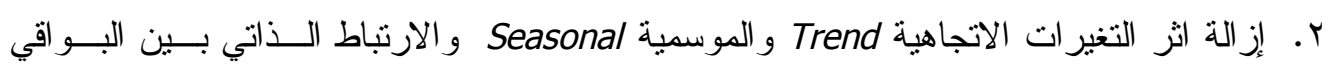
Autocorrelation متخذي القرار في رسم السياسات ووضع الخطط المستقلية لضمان استقرار الأسعار علي مدار

$$
\text { شهور السنة. }
$$

\section{الطريقة البحثية ومصادر البياتات}

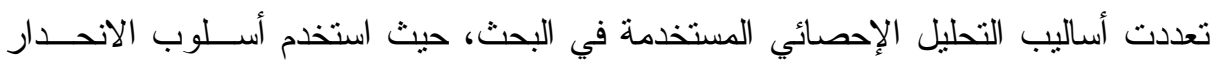

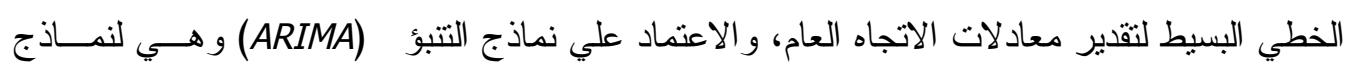

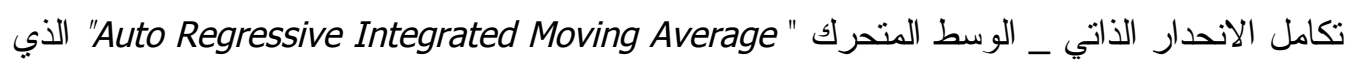
يعرف بنموذج بوكس- جينكيز 'Box-Jenkins وقد استخدمت بعض المعايير الإحصــائية لاختيــار

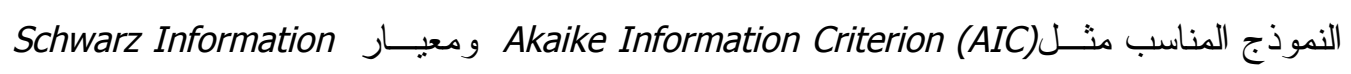

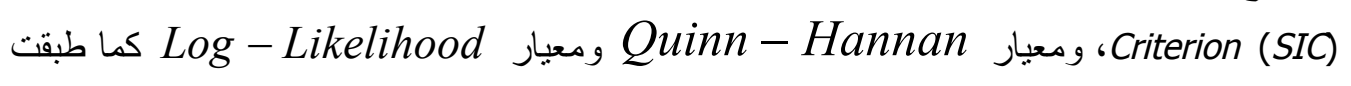

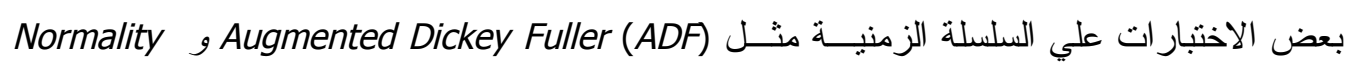
وذللك لاستبعاد اثر التغيرات الموسمية والاتجاهية و الارتباط الذاتي بين البواقي، كما تم الاعتماد

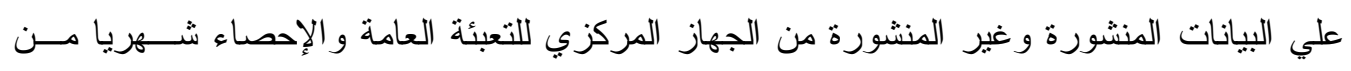

$$
\text { (r.17-r..人) }
$$

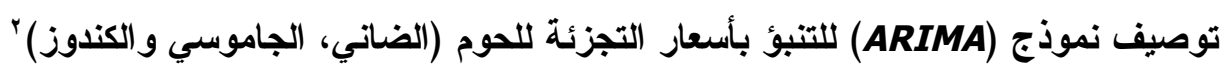

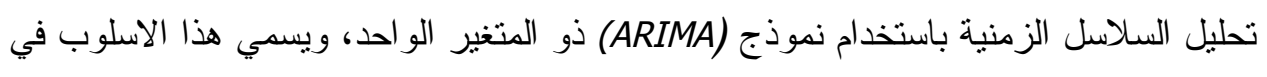

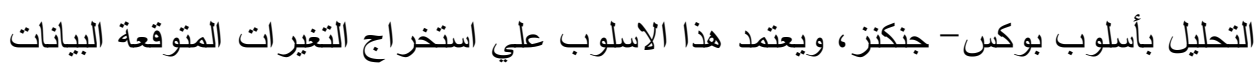

\footnotetext{
${ }^{1}$ Box, G. and Jenkins, G., (1976) "Time Series Analysis: Forecasting and Control", San Francisco. Calif, Holden- Day,U.S.A.

${ }^{2}$ Brock Well , P.J. and Davis, R.A. (1991), " Time Series Theory and Methods ",2nd ed, Springer Verlag New York Inc, New York.
} 
المشاهدة، وتتجز أ السلسلة الزمنية الي عدة مكونات أو عناصر تسمي ثلاثه مرشحات 'خطية هي

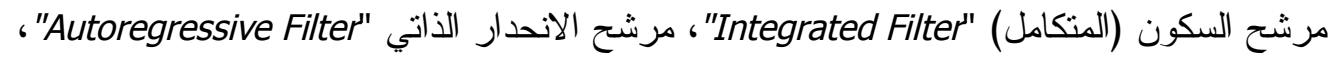
ومرشح المتوسطات المتحركة "Moving Avevage Filter" هو نموذج يعتمد كلية علي القيم الزمنية فقط للمتغير في التتبؤ ومن ثم يمكن تطبيقه علي أي متغير يتوفر عنه سلسلة زمنية طويلة نسبيا،

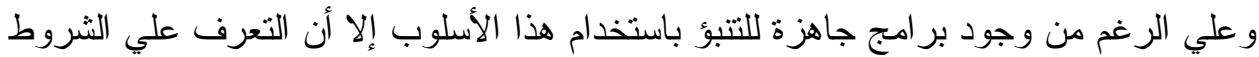
اللازمة لتطبيقه بطريقة صحيحة تضمن الحصول علي أفضل التقدير ات. ويتضمن نموذج (ARIMA)

\section{ت تلاث مرشحات}

(الأولي: مرشح السكون (المتكامل) Integrated Filter)

تحويل البيانات الغير ساكنة إلي بيانات ساكنة وذلك باستخدام اختبار جذر الوحسدة Unit root tests

ومن أهم اختبار ات التي تستخدم في جزر الوحدة اختبار (Augmented Dickey Fuller (ADF).

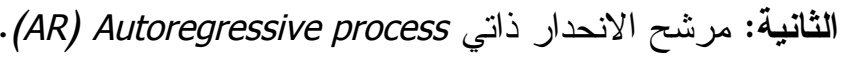

يعني أن المتغير التابع (Yt) في معادلة الانحدار الذاتي يكون دالة للقيم السابقة لهذا المتغير كما يلي: $Y_{t}=F\left(Y_{t-1}, Y_{t-2}, \ldots . ., Y_{t-p}\right)$

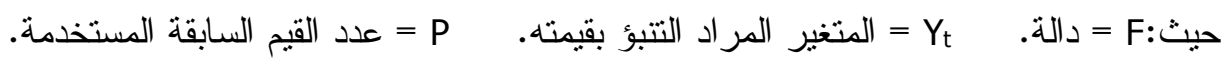
الثالثة: مرشح المتوسطات المتحركة Moving Average وتكون عن طريق جعل المتغير التابع (Yt) دالة للقيم السابقة لحد الخطأ Error Term كما يلي: $Y_{t}=F\left(E_{t-1}, E_{t-2}, \ldots ., E_{t-q}\right)$

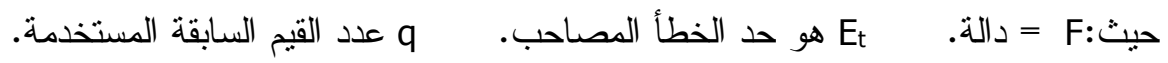
ولتكوين نموذج (ARIMA) من المعادلتين السابقتين يكون شكل المعادلة كما يلي:

$Y_{t}=B 0+\theta 1 Y_{t-1}+\theta 2 Y_{t-2}+\ldots \ldots \ldots . . . .+E \theta_{t-p}-\varepsilon_{t}-E \varphi_{t-2}, \ldots E \varphi a \varepsilon_{t-a}$ حبـثبثة $\quad$ Autoregressive Moving Average معاملات الانحدار الذاتي و المتوسطات المتحركة علي التزتيب، وقبل تطبيق المعادلة السـابقة علـي

' و الترفاندل (1999) (السلاسل الزمنية من الوجهة التطبيقية ونماذج بوكس -جنكنز ، دار المريخ للنشر، الرياض المملكة

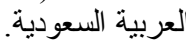

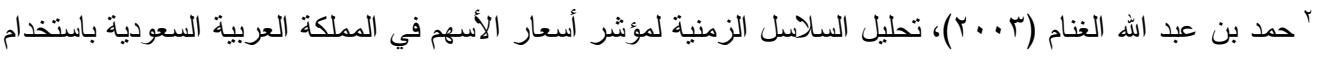

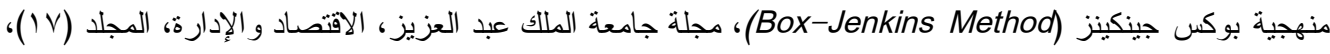

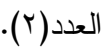

${ }^{3}$ Box, G. E. and Price, D. A. (1970), " Distribution of Residual Autocorrelations in Autoregressive - Integrated Moving Average Time Series Models ", JASA , Vol.55 , No.332, PP.1509-1525. 


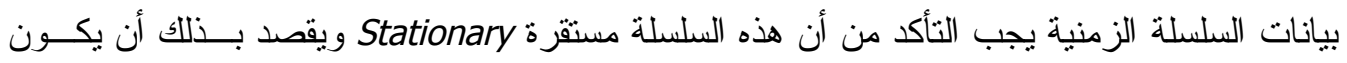

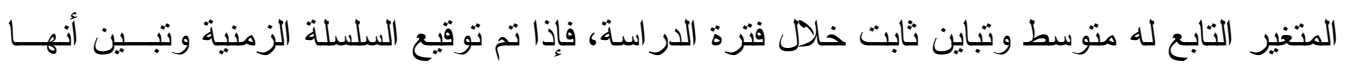

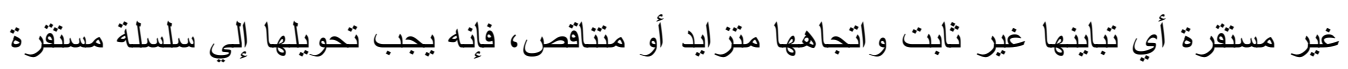
عن طريق إيجاد الفرق الأول d لهذا المتغير First Difference كما يلي: $Y_{t}^{*}=\Delta Y=Y_{t}-Y_{t-1}$

و إذا لم يترتب علي الفرق الأول سلسلة مستقرة يمكن أخذ الفرق الأول لهذا الفرق كما يلي: $-Y_{t-1}^{*}=\Delta Y-Y_{t-1}$ $Y_{t}^{* *}=\Delta Y_{t}^{*}=Y_{t}^{*}$

وبصفة عامة يمكن تكر ار عملية الفروق هذه عدة مرات حتي نحصل علي سلسلة مستقرة.

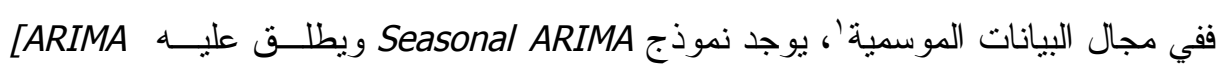
(p.d.q) S(p.d.q)]

\begin{tabular}{|c|c|}
\hline . Identification Stage & أولا: مرحلة التعريف \\
\hline Model Specification & ثانيا: مرحلة توصيف النموذج \\
\hline Estimation Stage & ثالثا: مرحلة تقدير معالم النموذج \\
\hline Diagnostic Stage & رابعا: مرحلة التشخيص \\
\hline Forecasting Stage & خامسا: مرحلة التنبؤ \\
\hline
\end{tabular}

معايير المفاضلة

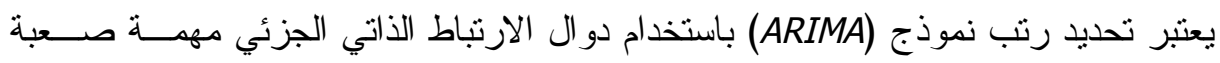

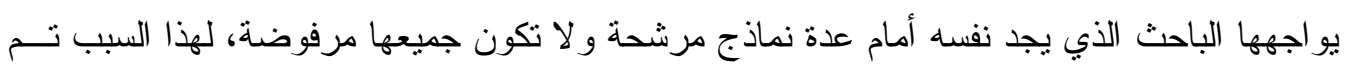

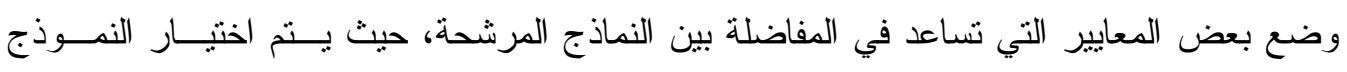
الأفضل الذي يملك اقل قيمة لهذه المعايير ومن هذه المعايير الإحصائية'؛

Schwarz information criterion ومعيار Akaike information criterion (AIC)

كلا المعيارين مبني على تباين البواقي

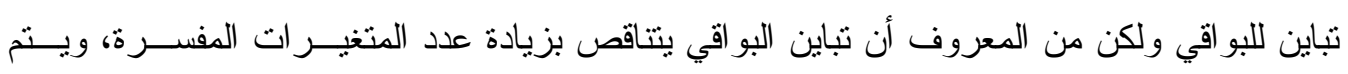
اختيار النموذج الذي يمنلاك اقل قيمة من AIC, SIC ، لأن السلسلة الزمنية تستخدم بأطو ال مختلفة يتن

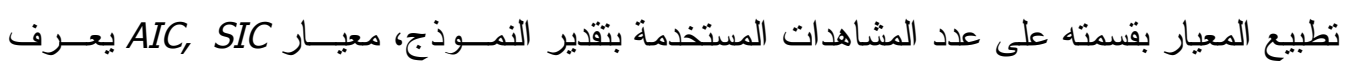
كالتالي:

' فاضل عباس الطائي، جيهاني فخري صالح الكور اني(1 . . ب)، التنبؤ بنماذج ARIMA الموسمية باستخدام طر ائق

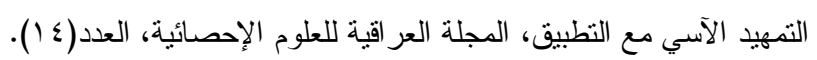

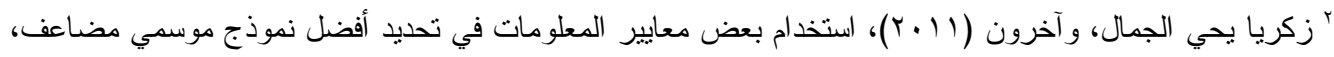
المجلة العر اقية للعلوم الإحصائية، العدد (9 (1). 


$$
\begin{gathered}
A I C=\ln (\overbrace{\sigma_{u}^{2}}^{-})+\frac{2 k}{n} \\
S I C=\ln (\overbrace{\sigma_{u}^{2}})+\frac{k \cdot \ln (n)}{n}
\end{gathered}
$$

معيار SIC يعتبر خيار بدلا من AIC لها نفس المعنى ولكن تعطي نقل لعدد المعاملات k لهذا السبب

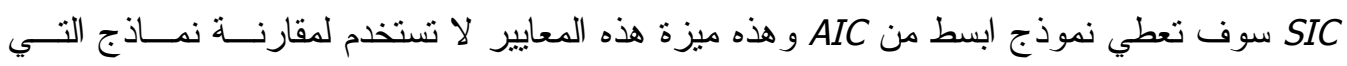

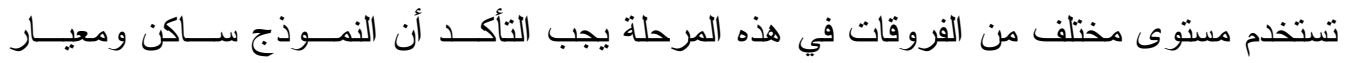
Log - Likelihood ومعيار Quinn - Hannan السلسلة الزمنية مثل Augmented Dickey Fuller (ADF) و Normality Test وذللك لاســتبعاد اثـر

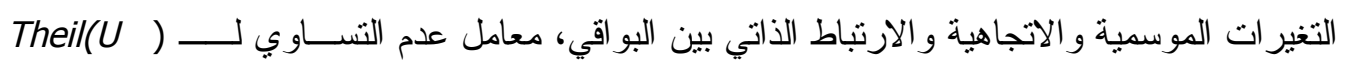

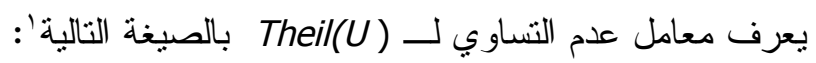

$$
U=\frac{\sqrt{\frac{1}{N} \sum_{t=1}^{N}\left(\hat{Y}_{t}-Y_{t}\right)^{2}}}{\sqrt{\frac{1}{N} \sum_{t=1}^{N}\left(\hat{Y}_{t}\right)^{2}+\sqrt{\frac{1}{N} \sum_{t=1}^{N}\left(Y_{t}\right)^{2}}}}
$$

حيث أن: N = عدد المشاهدات. $=$ = القيمة المقدرة للمتغير التابع. Y= = القيمة الفعلية للمتغير التابع. تتز اوح قيمة U بين الصفر و الو احد الصحيح، فإذا كانت 0 = المقدر علي التتبؤ بالو اقع خلال الفترة الزمنية للتقدير سوف تكون جيدة (له قدرة عالية علي التتبــؤ)، أما إذا كانت 1 كأن قدرة نموذج الانحدار المقدر علي النتبؤ بالو اقع خلال الفترة الزمنية للتقدير سوف تكون سيئة(عدم قدرة علي النتبؤ).

\section{تعليل التغير الموسمي}

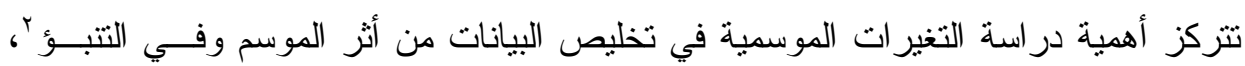

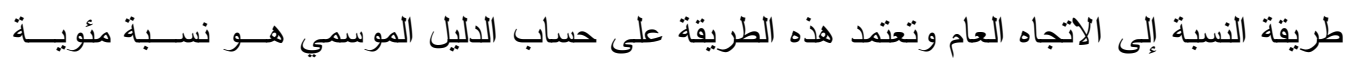
توضح أثر الموسم في الظاهرة محل الدراسة.

$$
\begin{aligned}
& s_{i}=\frac{m_{i}}{\sum m_{i}} 100 \\
& \text { الدليل الموسمي لكل موسم }=S_{i}
\end{aligned}
$$

$$
\text { الدليل الموسي = }
$$

$$
\text { حيث: }
$$

${ }^{1}$ Blieme .F,(1973) "Theils Forecast Accuracy Coefficient: A Clarification" Journal of Marketing Research,Vol.X (November), 444-446.

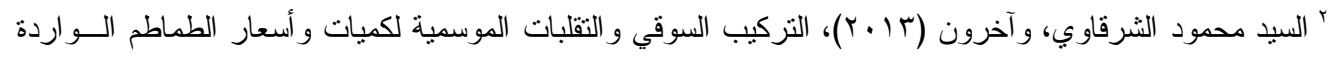

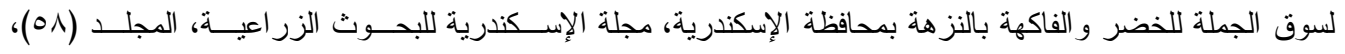




\section{التطور الموسمي لأسعار لحوم جاموسي صغير السن:}

يوضح جدول (1) موسمية أسعار لحوم جاموسي صغير السن بنلو بالعظم في مصر و الذي لني

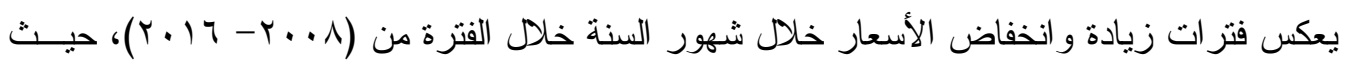

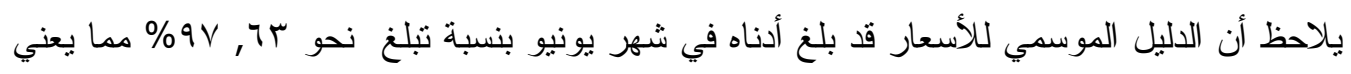

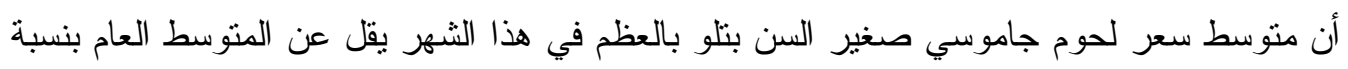

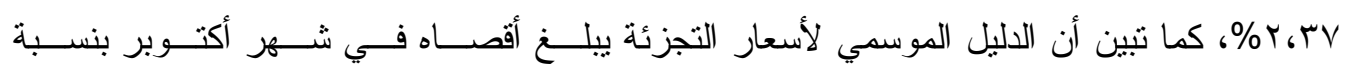

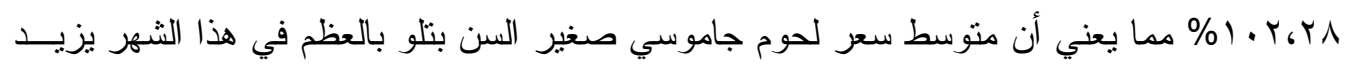

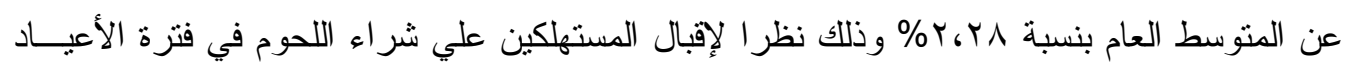

$$
\begin{aligned}
& \text { (عيد الاضحي المبارك و ر أس السنة الهجرية). } \\
& \text { أو لا نوضيح الاسباب : }
\end{aligned}
$$

\begin{tabular}{|c|c|c|}
\hline نسبة التغير (\%) & الاليل الموسمي & الشهور \\
\hline . $\vee$ A - & $99,4 \mathrm{~T}$ & يناير \\
\hline • ‘৯৭- & 99611 & فبر اير \\
\hline $1,7 \cdot-$ & $9 \Lambda_{6} \varepsilon$. & مارس \\
\hline $.6 \times 7-$ & $99.4 \leq$ & أبريل \\
\hline $16 \times 9-$ & $9 \wedge, 4)$ & مايو ل \\
\hline T.rV & 9V.74 & يونيو \\
\hline $1.0 \mathrm{~V}-$ & $9 \wedge, \varepsilon r$ & يوليه \\
\hline I.rT & $1.16 \mathrm{~K}$ & أغسطس \\
\hline 1.67 & 1.1674 & سبتمبر \\
\hline r.rA & I. T.YA & أكتوبر \\
\hline tort & D. & نوفمبر \\
\hline r.rT & $1 . T_{6} Y_{7}$ & ديسمبر \\
\hline- & $1 \cdot r, \varepsilon r$ & لمتوسط العام \\
\hline
\end{tabular}

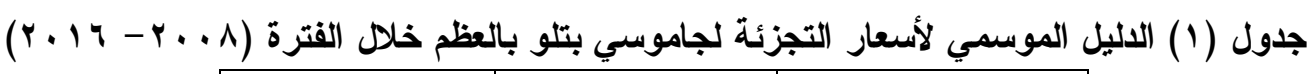

المصدر : جمعت وحسبت من الجهاز المركزي للنعبئة العامة والإحصاء، النشرة الثهرية لمتوسط أسعار المستهلك لأهم السلع الغذائية، أعداد مختلفة. ثانيا التطور الموسمي لأسعار لحوم كندوز أغلاد

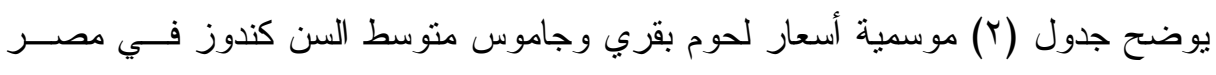

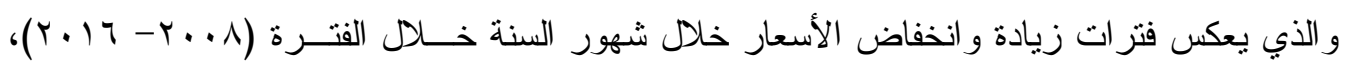

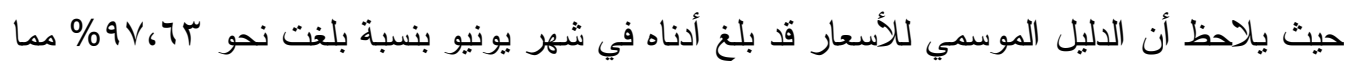

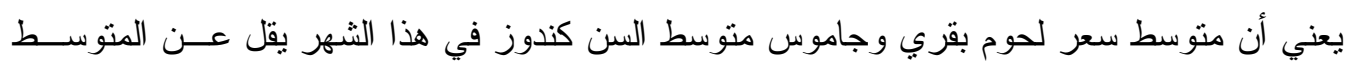

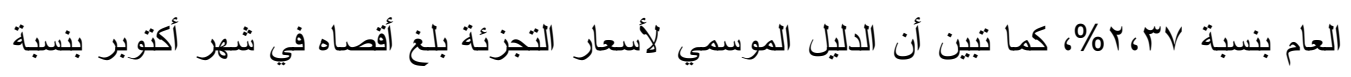

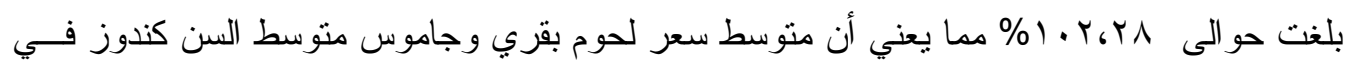

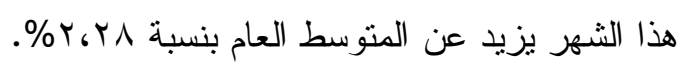




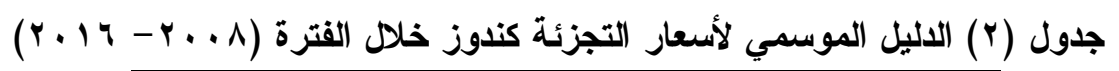

\begin{tabular}{|c|c|c|}
\hline نسبة التغير (\%) & اللاليل الموسمي & الشهور \\
\hline$-\cdot .7 \leqslant$ & 99.47 & يناير \\
\hline - •، & $99.1 \mathrm{~V}$ & فبر اير \\
\hline-1 .0r & $9 \Lambda_{6} \leqslant V$ & مارس \\
\hline$-1,41$ & 91.74 & أبريل \\
\hline$-1,10$ & QA, YO & مايو \\
\hline$-T_{6} \leqslant r$ & $9 V_{6} 0 \mathrm{~V}$ & يونيو \\
\hline$-1,20$ & 91.10 & يوليه \\
\hline$-\cdot ، \varepsilon$. & 99.7. & أغسطس \\
\hline 1.77 & 1.1 .77 & سبتمبر \\
\hline r.rT & I. T.r. & أكتوبر \\
\hline T.VT & $1 . r_{6} V r$ & نوفمبر \\
\hline$\varepsilon v, r$ & $\varepsilon v, 1, r$ & ديسمبر \\
\hline- & $r v, 1.1$ & لمتوسط العا \\
\hline
\end{tabular}

المصدر : جمعت وحسبت من الجهاز المركزي للنعبئة العامة والإحصاء، النشرة الثهرية لمنوسط أسعار المستهلك لأهم

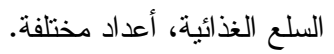

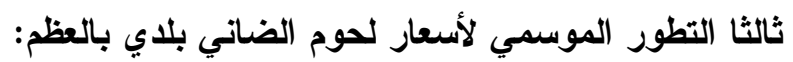

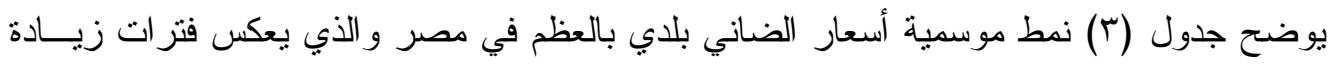

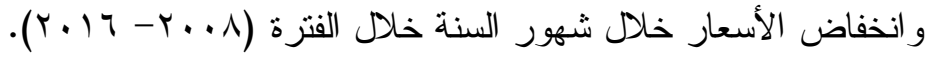

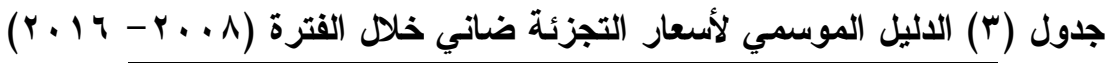

\begin{tabular}{|c|c|c|}
\hline نسبة التغير (\%) & الدليل العوسمي & الثهور \\
\hline $1,7.7-$ & $9 \wedge, 9 \leqslant$ & يناير \\
\hline$\cdot, 7 \cdot-$ & $99, \leqslant$. & فبراير \\
\hline$\cdot, V \leqslant-$ & 99,47 & مارس \\
\hline $1, Y Y-$ & $q \wedge, \vee \wedge$ & أبريل \\
\hline $1, \leqslant Y-$ & $9 \wedge, 0 \wedge$ & مايو \\
\hline$r, \cdot q-$ & $9 \vee, 9)$ & يونيو \\
\hline $1, \leqslant \%-$ & $9 \Lambda,, 0 V$ & يوليه \\
\hline$\cdot,^{r}-$ & 99,79 & أغسطس \\
\hline 1, & 1. 1, 1, & سبتمبر \\
\hline$r_{,}, \varepsilon$ & $1 \cdot r, \cdot \varepsilon$ & أكتوبر \\
\hline$r, 7 q$ & $1 \cdot r, 79$ & نوفمبر \\
\hline$r, O r$ & $1 \cdot r, 0 r$ & لديسمبر \\
\hline - & $\cdot v, 1 \cdot r$ & لمتوسط العام \\
\hline
\end{tabular}

المصدر : جمعت وحسبت من الجهاز المركزي للنعبئة العامة والإحصاء، النشرة الثهرية لمتوسط أسعار المستهلك لأهم لفام

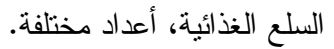

حيث يلاحظ أن الدليل الموسمي للأسعار قد بلغ أدناه في شهر يونيو بنســبة بلغـــت نحــو

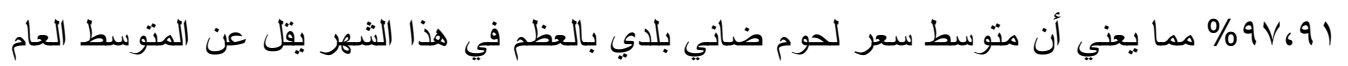

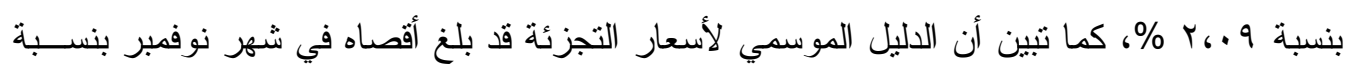




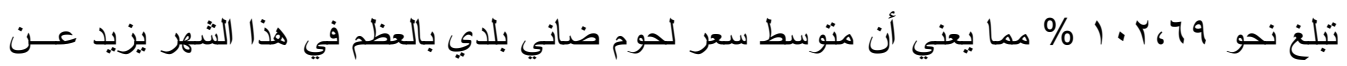

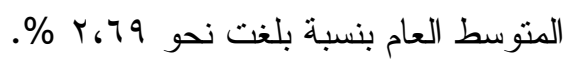

تقدير أسعار لحوم الجاموسي صغير السن:

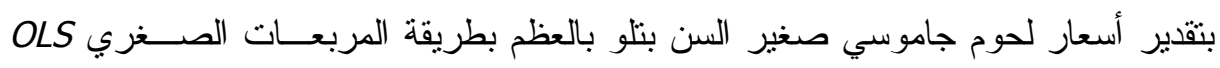

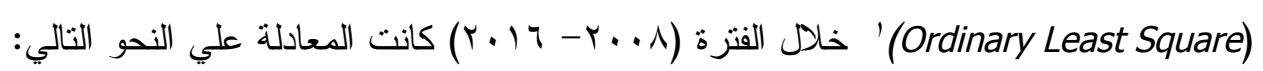
$Y_{i}=\underset{(31.55)^{* *}}{26.32}+\underset{(51.99)^{* *}}{0.69} X_{i}$

$$
\begin{aligned}
& R=0.98 \quad R^{2}=0.96 \quad D W=0.15 \\
& \text { حيث أن: } \\
& \text { Log - Likelihood }=-309.24 \\
& \text { Akaike Criterion }=622.47
\end{aligned}
$$

Schwarz Criterion $=627.84$

Quinn - Hannan $=624.65$

تشير المعادلة السابقة إلى أن أسعار لحوم جاموسي صغير السن بتلو بالعظم تزيــد ســنـياً

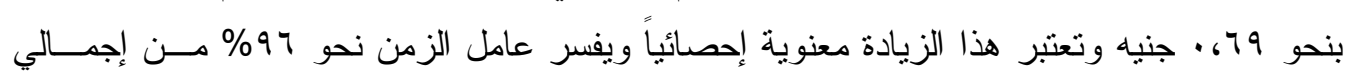

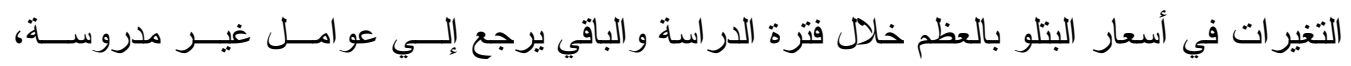

\begin{tabular}{|c|c|c|c|c|}
\hline$r \cdot r l$ & $r \cdot r \cdot$ & $r .19$ & $r \cdot 1 \Lambda$ & الثهور \\
\hline $1 \pi \leqslant, 70$ & $M \pi, r v$ & $111, \cdot 9$ & $1 \cdot 9, \wedge 1$ & يناير \\
\hline $1 r 0, r \varepsilon$ & $I Y V, \cdot T$ & $\| \wedge, \vee \wedge$ & $11 \cdot, 0$ & فبر اير \\
\hline r & Irv, vo & $119, \leqslant v$ & 111,19 & مارس \\
\hline $1 T_{1}, \mathrm{r}$ & $\mid r \wedge, \varepsilon \varepsilon$ & $1 r_{0}, 17$ & $111, \wedge \Lambda$ & أبريل \\
\hline$|r v, \varepsilon|$ & $1 r q, 1 r$ & $M \cdot, \lambda^{\circ}$ & $\| r, O V$ & مايو \\
\hline $1 r \wedge, 1$ & $M q, \wedge r$ & $|r|, 0 \leqslant$ & $11 r, r_{4}$ & يونيو \\
\hline $1 r \Lambda, \vee q 9$ & $1 \pi \cdot, 01$ & $M r, r T$ & 115,90 & يوليه \\
\hline $1 \pi q, \varepsilon 1$ & $1 \pi, r$ & IrT, Gr & $11 \varepsilon, 7 \varepsilon$ & أغسطس \\
\hline $1 \leqslant \cdot, I V$ & $1 \pi 1, \wedge 9$ & $|r r, T|$ & r & سبتمبر \\
\hline $1 \leqslant \cdot, \wedge T$ & $1 r r, O 1$ & $\mid r \varepsilon, r$ & $117, \cdot r$ & أكتوبر \\
\hline $1 \leqslant 1,00$ & IrT, rV & $\mid r \varepsilon, 99$ & 117,51 & نوفمبر \\
\hline $1 \leq r, r \leq$ & $1 \pi r, 97$ & $\mid r_{0}, T \Lambda$ & $11 v_{1} \varepsilon$ & ديسمبر \\
\hline
\end{tabular}

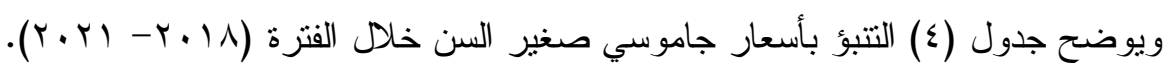
جدول ( ؛ ) التنبؤ بأسعار لحوم جاموسي صغير صنير السن

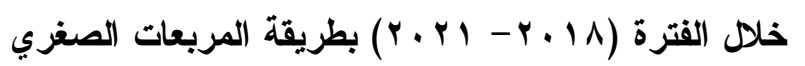

Gret/ المصدر : حسبت باستخدام برنامج

' إبر اهيم سليمان، و آخرون (10 ب ( )، مقدمة في الاقتصاد القياسي، الطبعة الأولي، الككتبة الأكاديمية. 
Stationouity Test اختبار الاستقر ارية

تشبير قبية DW و المقدرة بنحو 10 ، • إلي وجود ارتباط ذاتي بين البــو اقي فــي السلســلة

الزمنية مما يجعلها في حالة عدم السكون، كما نم إجر اء اختبـار جـذر الوحــة (Unit root Test) للتعرف علي درجة تكامل السلسلة الزمنية للمتغيرات الاقتصادية محل الدراسة لـعرفة ما إذا كانــــ

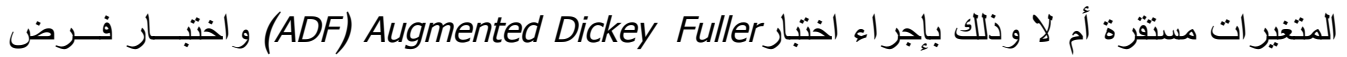

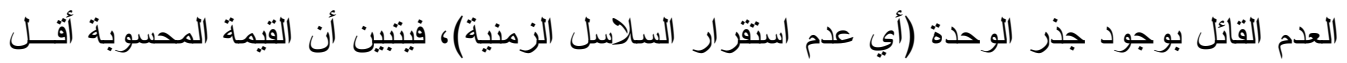
من القيمة الجدولية وهذا يدل علي وجود جذر الوحدة أي أن السلسلة غير ساكنة أو غيـر مســتقرة و السبب وجود اتجاه عام عشو ائي مما يستوجب معالجة السلسلة قبل التتبؤ لزيادة دقة التتبؤ بأســعار لحوم جاموسي صغير السن بنلو بالعظم. أكدت قيم معاملات الارنباط الذاتي و الجزئي كما هو موضح بالثكل (1) و التي أظهرت فيه

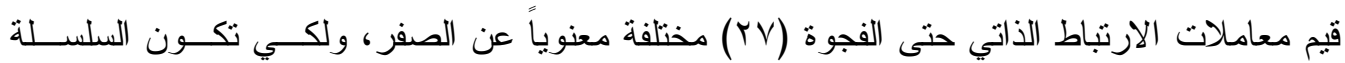

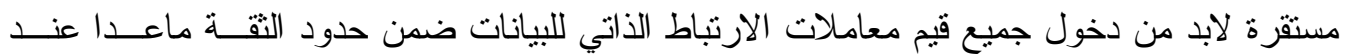

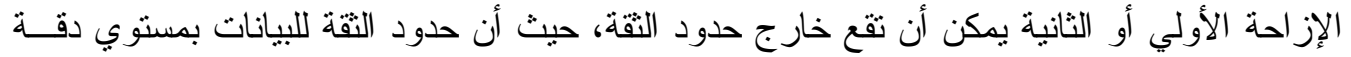

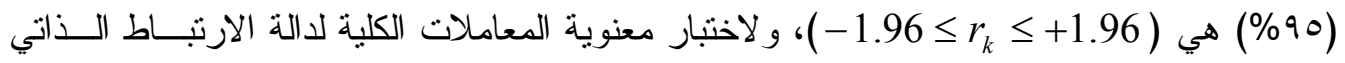
(Q.stat) Ljung\& Box ماستخدام

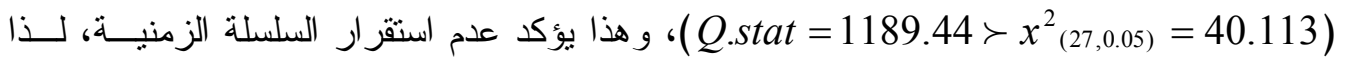

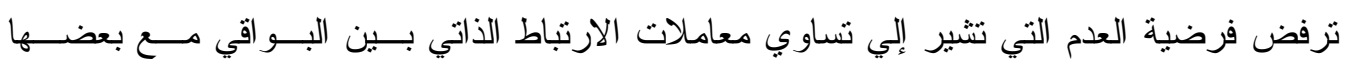
ومساوتها بالصفر ، وتقبل الفرضية البديلة وهذا يعني أن السلسلة الزمنية غير مستقرة.

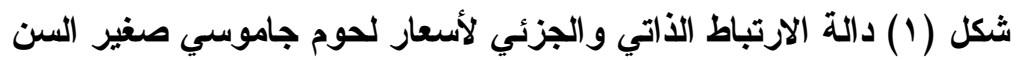
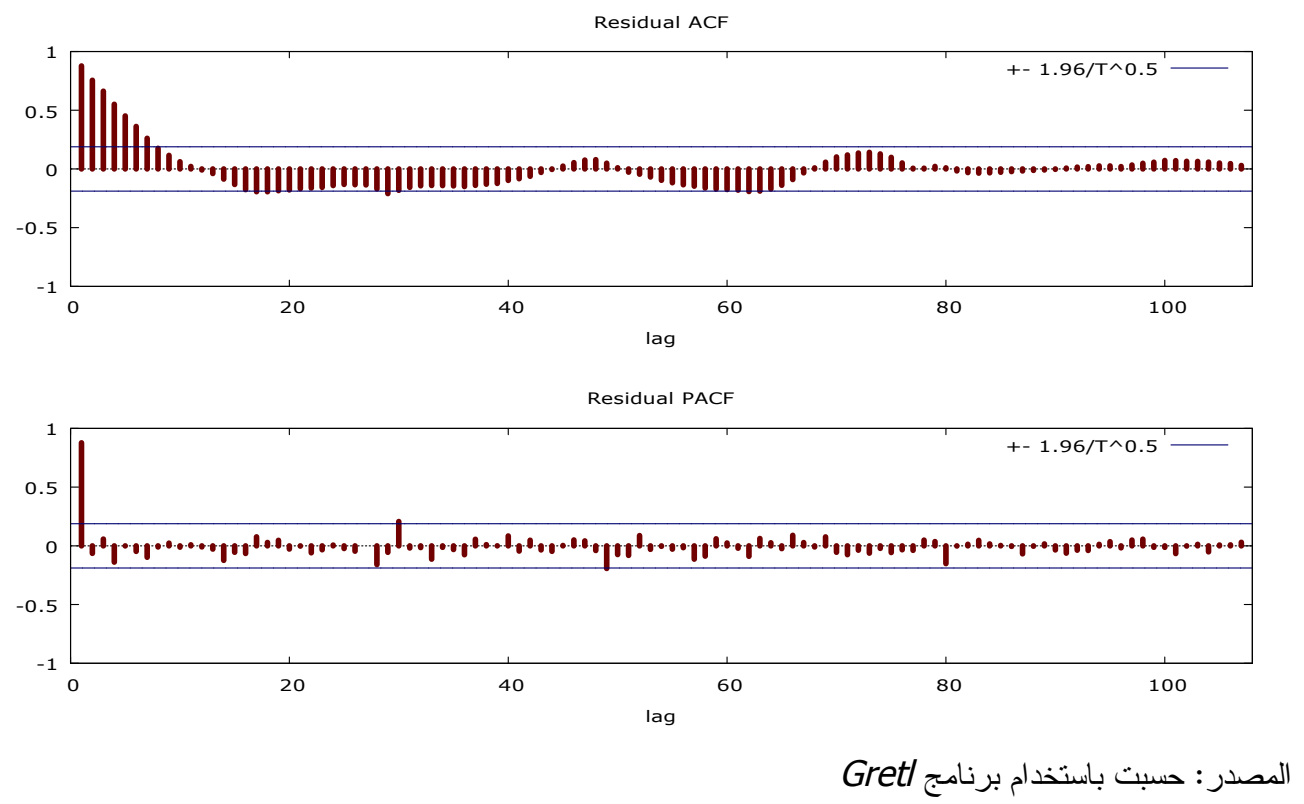
تقدير أسعار لحوم كندوز:

تقدير أسعار لحوم بقري وجاموس متوسط السن كندوز بطريقة المربعات الصغري

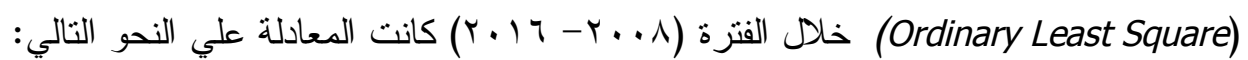
$Y_{i}=\underset{(45.87)^{* * *}}{31.06}+\underset{(57.06)^{* * *}}{0.62} X_{i}$

$R=0.98 \quad R^{2}=0.97 \quad \begin{array}{ll}D W & =0.14 \\ & \end{array}$

Log - Likelihood $=-287.35$

Akaike Criterion $=578.71$

Schwarz Criterion $=584.07$

Quinn - Hannan = 580.88

تشير المعادلة السابقة إلى أن أسعار لحوم بقري وجاموس متوسط السن كندوز تزيد ســنوياً

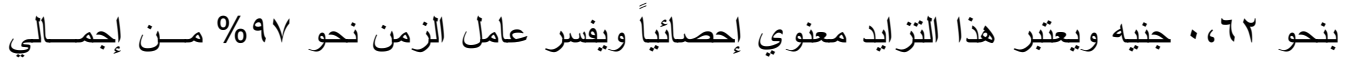

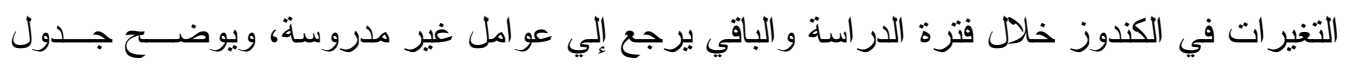

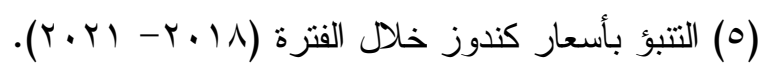

Stationouity Test اختبار الاستقرارية

تشبير قبيمة DW و المقدرة بنحو ـ (، • إلي وجود ارتباط ذاتي بين البــو اقي فــي السلســلة

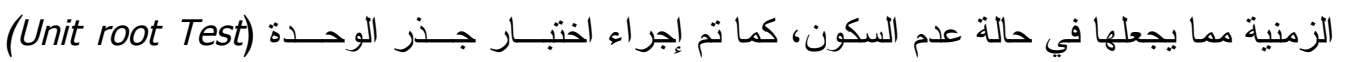

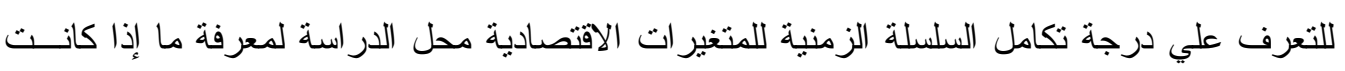

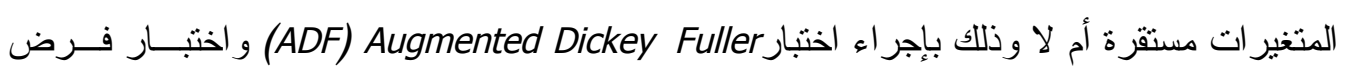

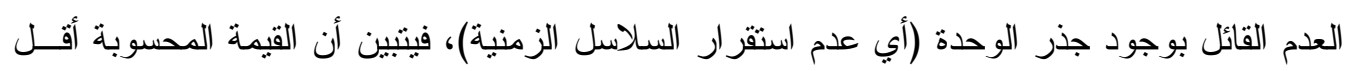

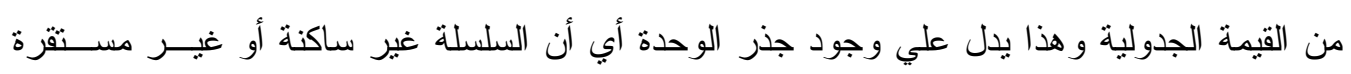

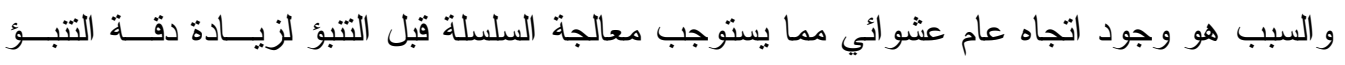

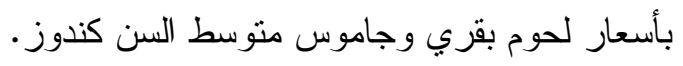
جدول (0) التتبؤ بأسعار لحوم كندوز

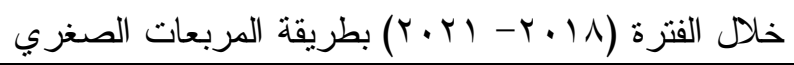

\begin{tabular}{|c|c|c|c|c|}
\hline$Y \cdot Y I$ & $r \cdot r \cdot$ & 5.19 & $r \cdot 1 \Lambda$ & الثهور \\
\hline$T Y \Lambda, \varepsilon$. & $1 Y \cdot, 97$ & $11 r, 0 r$ & $1 \cdot 7,11$ & يناير \\
\hline $1 r q, \cdot r$ & $\mid Y_{1}, 01$ & $11 \leq, 1 \leq$ & $1 \cdot 7,7$. & فبر اير \\
\hline $1 Y q, 7 \leq$ & $I r r, r$. & $11 \leq, \sqrt{ } \times$ & I.V,r & مارس \\
\hline $1 \mu_{\bullet}, r_{4}$ & $M r, \wedge r$ & $110, \pi \wedge$ & $1 \cdot v, 9 \leq$ & آبريل \\
\hline $1 T \cdot, \lambda \Lambda$ & $\mid r r, \varepsilon \varepsilon$ & $117, \ldots$ & $1 \cdot 1,07$ & مايو \\
\hline $1,1,0$. & $\mid Y \varepsilon, \cdot T$ & 117,74 & $1.9,11$ & يونيو \\
\hline ITr,IT & $\mid r \varepsilon, 7 \wedge$ & $\| V, r \varepsilon$ & $1.9,1$. & يوليه \\
\hline$I T, V \varepsilon$ & $\mid r 0, r$. & $11 \vee, \wedge 7$ & $11 \cdot, \leqslant r$ & أغسطس \\
\hline דז,ז"ו & $1 r 0,9 r$ & $\| 1, \leqslant \Lambda$ & $111, \cdot \varepsilon$ & سبتمبر \\
\hline 1ז,9זו & $1 \times 7,0 \leq$ & $119,1$. & 111,77 & أكتوبر \\
\hline $1 \pi \varepsilon, 7$. & $1 Y v, 17$ & $119, \mathrm{VT}$ & $\| r, r \wedge$ & نوفمبر \\
\hline $1 T 0, Y Y$ & IYV,VA & $M \cdot r \varepsilon$ & $11 r, 9$. & ديسمبر \\
\hline
\end{tabular}

Gret/ المصدر : حسبت باستخدام برنامجر 
أكدت قيم معاملات الارتباط الذاتي و الجزئي كما هو موضح بالثكل (Y) و التي أظهرت فيه

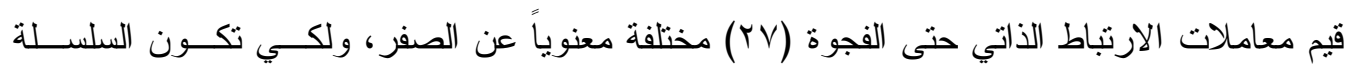

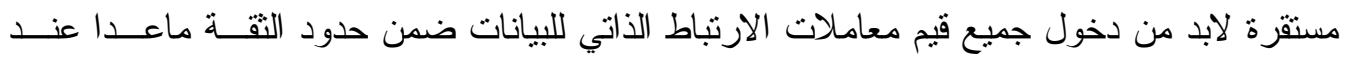

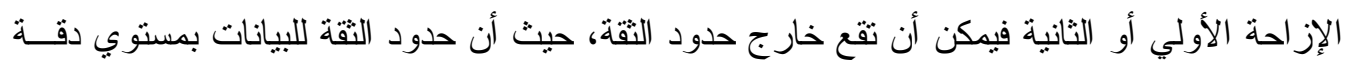

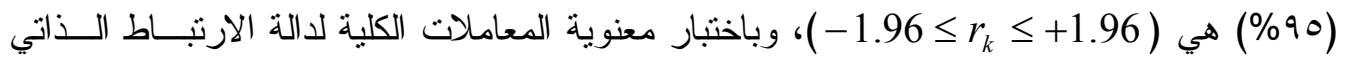
(Q.stat) Ljung\& Box ماستخدام

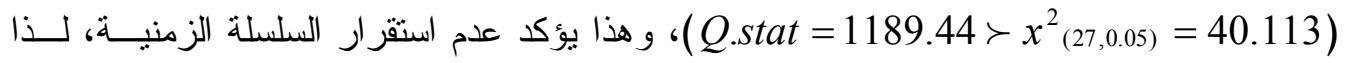

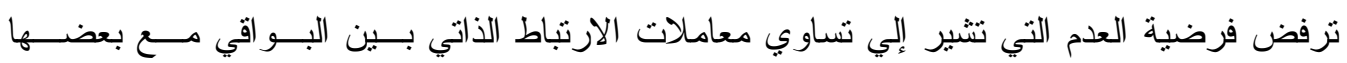
ومساوتها بالصفر ، وتقبل الفرضية البديلة وهذا يعني أن السلسلة الزمنية غير مستقرة. شكل (r) دالة الارتباط الذاتي و الجزئي لأسعار لحوم كندوز

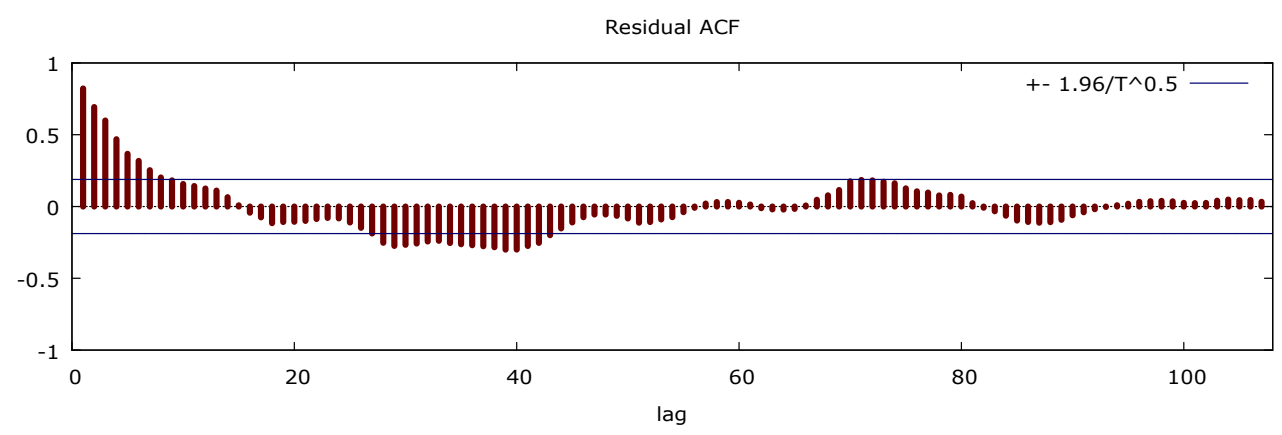

Residual PACF

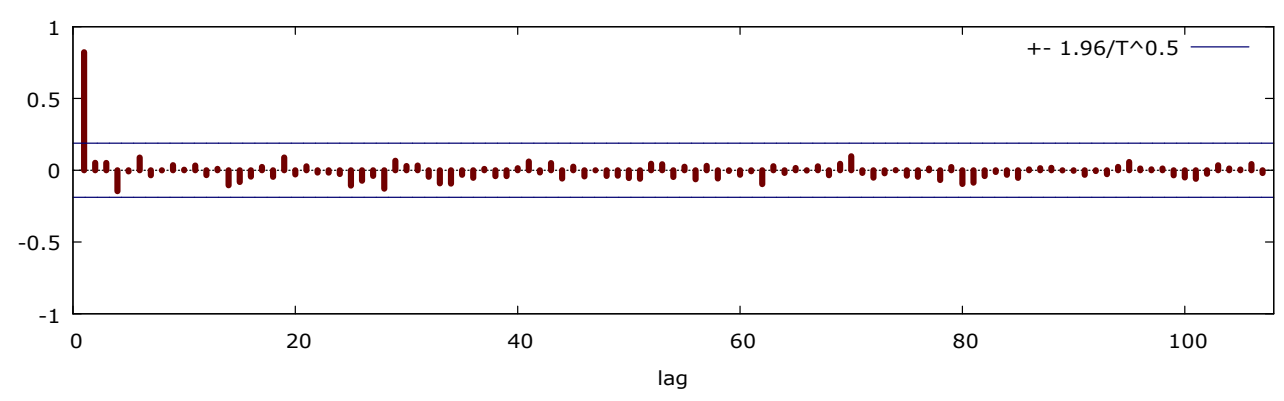

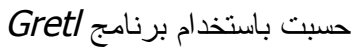

تقدير أسعار لحوم ضاني بلاي

Oتقدير أسعار لحوم ضاني بلدي بطريقة المربعات الصغري OLS (Square $Y_{i}=\underset{(48.17)^{* * *}}{30.49}+\underset{(64.67)^{* *}}{0.65} X_{i}$

$R=0.99 \quad R^{2}=0.98 \quad D W=0.16$

Log - Likelihood $=-280.08$

Akaike Criterion $=564.16$ 
Schwarz Criterion $=569.52$

Quinn - Hannan = 566.33

تشير المعادلة السابقة إلى أن أسعار لحوم ضاني بلدي تزيد سنوياً بنحو 70، · جنيه ويعتبر

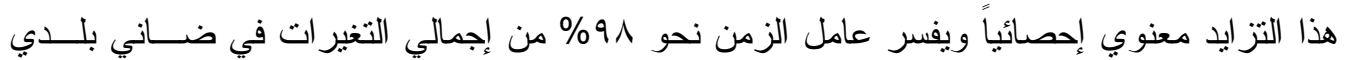

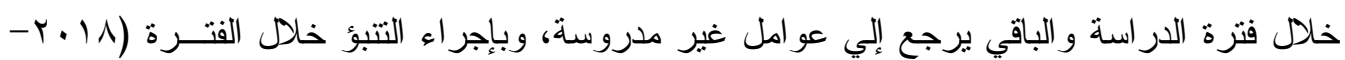

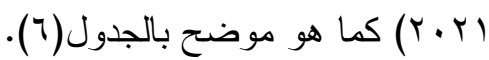

\section{Stationouity Test اختبار الاستقرارية}

تشير قيمة DW و المقدرة بنحو 7 ا، • إلي وجود ارتباط ذاتي بين البــو اقي فـي السلســلة الزمنية مما يجعلها في حالة عدم السكون، كما تم إجر اء اختبــار جـذر الوحسدة (Unit root Test)

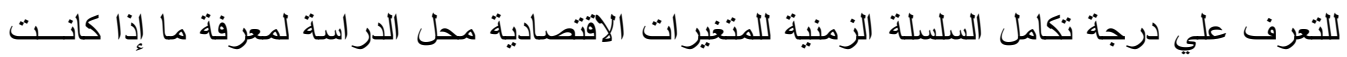

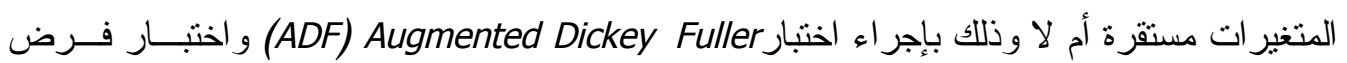
العدم القائل بوجود جذر الوحدة (أي عدم استقر ار السلاسل الزمنية)، فيتبين أن القيمة المحسوبة أقــلـل

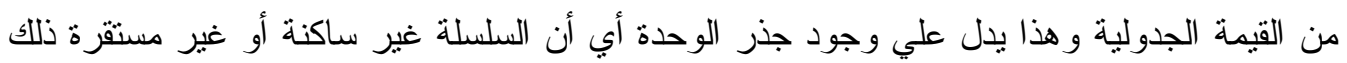

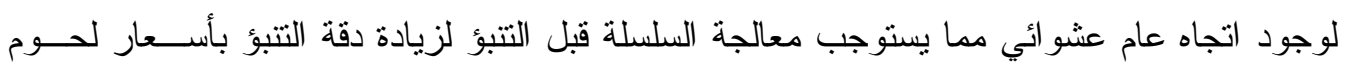
ضاني بلدي. جدول (†) التبؤ بطريقة المربعات الصغري أسعار لحوم ضاني بلدي

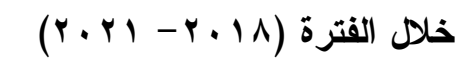

\begin{tabular}{|c|c|c|c|c|}
\hline$r \cdot r)$ & $r \cdot r \cdot$ & $r .19$ & $r \cdot 11$ & الثهور \\
\hline ST,O & $M \varepsilon, V \varepsilon$ & $117,9 \leq$ & $1.9,1 \leq$ & يناير \\
\hline 9 & $1 r_{0}, r_{q}$ & 118,09 & $1.9, \times 9$ & فبر اير \\
\hline & $\mid K 4, \cdot \varepsilon$ & $\| \wedge, r \varepsilon$ & $11 \cdot, \varepsilon \leq$ & مارس \\
\hline $1 \pi \leq, \leq 9$ & Irצ,79 & $11 \wedge, \wedge 9$ & $111, .9$ & أبريل \\
\hline $\mid \tau 0,1 \varepsilon$ & $\mid r V, r \varepsilon$ & $119,0 \varepsilon$ & $111, v \varepsilon$ & مايو \\
\hline Iro,vq & $\mid r v, q 9$ & $1 r_{\cdot}, 19$ & וr,rq & يونيو \\
\hline $\mid M_{4}, \varepsilon \varepsilon$ & $\mid r \wedge, T \varepsilon$ & $\mathbb{T} \cdot, \wedge \varepsilon$ & $\| r, \cdot \varepsilon$ & يوليه \\
\hline $1 \pi v, \cdot 9$ & $1 r q, r q$ & $|r|, \leqslant q$ & 114,79 & أغسطس \\
\hline $1 r v, v \varepsilon$ & $1 Y q, q \leq$ & $|K r,| \varepsilon$ & $\| \leqslant, r \leqslant$ & سبتمبر \\
\hline IrN,rq & $1 r \cdot, 09$ & Irr,vq & $11 \leqslant, 99$ & أكتوبر \\
\hline $1 \% q, \cdot \varepsilon$ & $|r|, r \varepsilon$ & $\mid r r, \varepsilon \varepsilon$ & $110,7 \leqslant$ & نوفمبر \\
\hline 149,79 & (r), & $\mid r \varepsilon, \cdot 9$ & $117, r 9$ & ديسمبر \\
\hline
\end{tabular}

Gret/ المصدر :حسبت باستخدام برنامبر

أكدت قيم معاملات الارتباط الذاتي و الجزئي كما هو موضح بالثكل (r) و التي أظهرت فيه

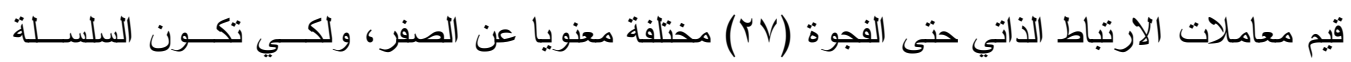

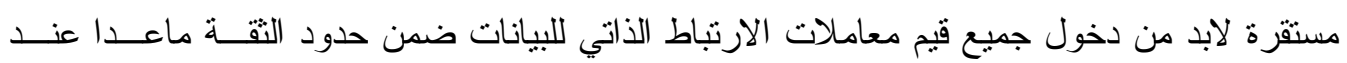

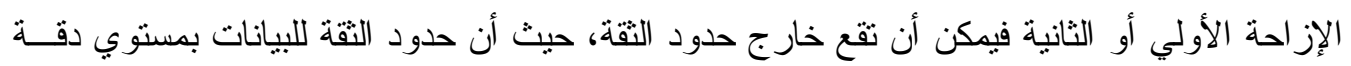


(\%0\%) هي (1.96 باستخدام (Q.stat) Ljung\& Box

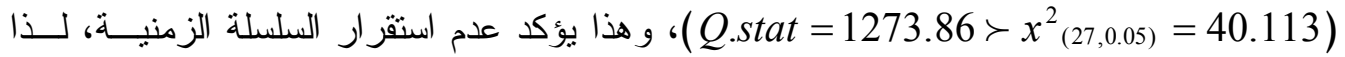

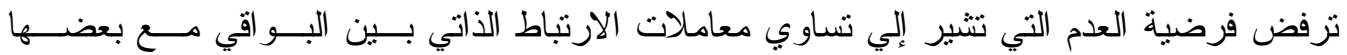
ومساوتها بالصفر، وتقبل الفرضية البديلة وهذا يعني أن السلسلة الزمنية غير مستقرة.

شكل (r) دالة الارتباط الاتاتي والجزئي لأسعار لحوم ضاني بلاي
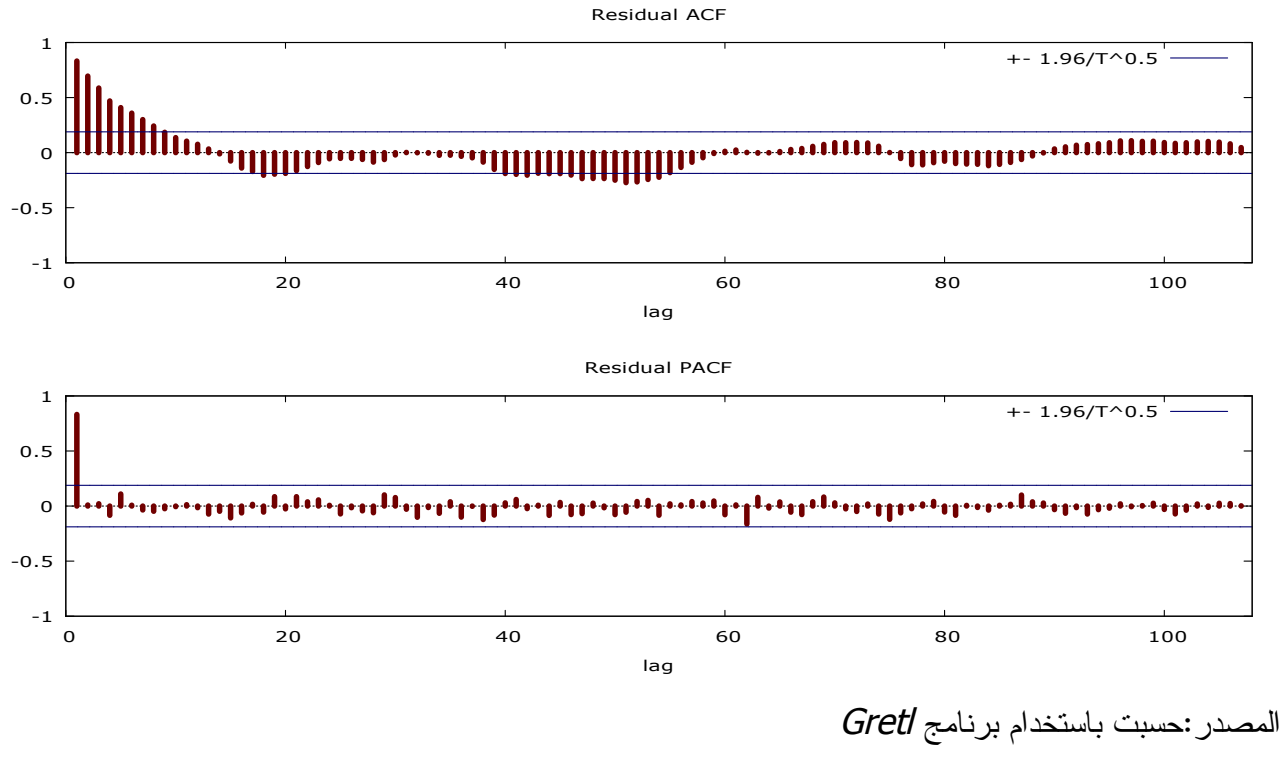

التتبؤ بأسعار لحوم جاموسي صغير السن

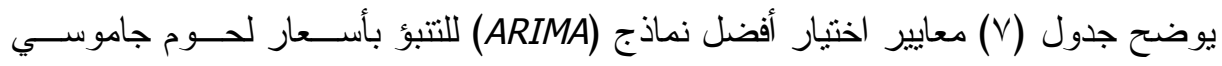

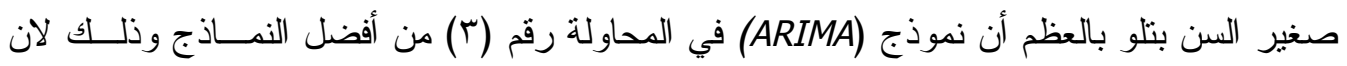

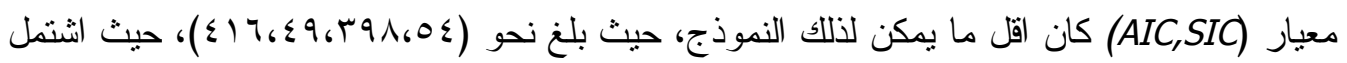

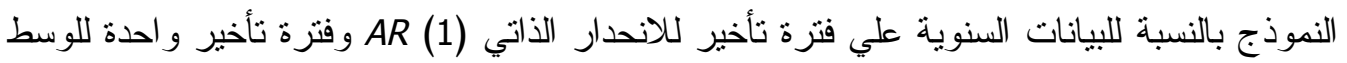

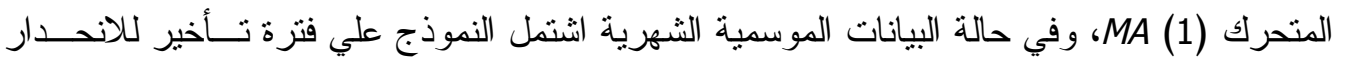

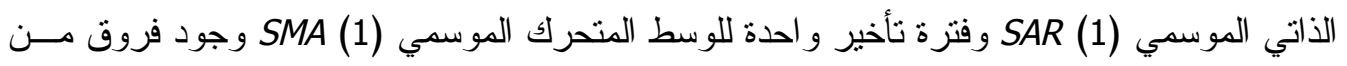
الرتبة الأولي بين شهور الفترة موضع الدر اسة. 
جدول (V) معايير المفاضلة بين نماذج (ARIMA) للتنبؤ بأسعار لحوم جاموسي صغير السن

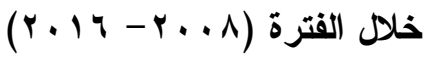

\begin{tabular}{|c|c|c|c|c|c|c|c|c|c|c|}
\hline \multirow{3}{*}{ Trying } & \multicolumn{7}{|c|}{ ARIMA Model } & \multicolumn{7}{c|}{ Model Selection } \\
\cline { 2 - 13 } & \multicolumn{3}{|c|}{ Non Seasonal } & \multicolumn{3}{c|}{ Seasonal } & \multicolumn{1}{c|}{} \\
\cline { 2 - 12 } & $P$ & $D$ & $Q$ & $P$ & $D$ & $Q$ & AIC & SIC & LL & $Q H$ \\
\hline 1 & 1 & 0 & 2 & 1 & 1 & 1 & 400.48 & 420.99 & 192.24 & 408.78 \\
\hline 2 & 2 & 0 & 1 & 2 & 1 & 1 & 401.46 & 424.54 & 191.73 & 410.79 \\
\hline 3 & 1 & 0 & 1 & 1 & 1 & 1 & 398.54 & 416.49 & 192.27 & 405.80 \\
\hline 4 & 1 & 0 & 2 & 1 & 1 & 2 & 402.19 & 425.27 & 192.10 & 411.52 \\
\hline 5 & 1 & 0 & 1 & 1 & 0 & 1 & 417.42 & 436.20 & 201.71 & 425.04 \\
\hline
\end{tabular}

ويوضح جدول (^) نتائج تقدير المحاولة رقم (؟) لنموذج (ARIMA) للنتبؤ بأســعار لحسوم

جاموسي صغير السن بتلو بالعظم.

جدول (^) نموذج (ARIMA) للتنبؤ بأسعار لحوم جاموسي صغير السن بتلو بالعظم

\begin{tabular}{|c|c|c|}
\hline Model & Beta & Z-test \\
\hline Constant & 0.027 & $0.015^{-}$ \\
AR1 & 0.960 & $19.95^{\circ *}$ \\
SAR2 & $0.149-$ & $1.2-^{-}$ \\
MA1 & 0.208 & $1.87^{\circ}$ \\
SMA 2 & $1.00-$ & $5.48-\wedge^{\circ}$ \\
SI & 0.738 & $4.39^{\circ *}$ \\
\hline
\end{tabular}

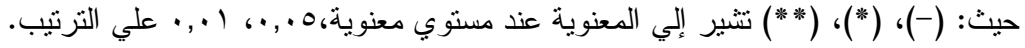

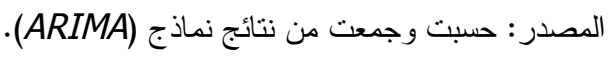

جدول (9) التتبؤ بأسعار لحوم جاموسي صغير السن بعد معالجة السلسلة

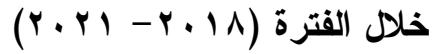

\begin{tabular}{|c|c|c|c|c|c|c|c|c|c|c|c|c|}
\hline \multicolumn{3}{|c|}{ r.rI } & \multicolumn{3}{|c|}{ r.r. } & \multicolumn{3}{|c|}{ r.19 } & \multicolumn{3}{|c|}{ r.1A } & \multirow[t]{2}{*}{ الشهور } \\
\hline حد أعلي & التنبؤُ & حد أدني & حد أعلي & التنبؤر & حد أدني & حد أعلي & التنبؤر & حد أدني & حد أعلي & التتبؤُ & حد أدني & \\
\hline $10 \%, 01$ & $|\leqslant|, \wedge \mid$ & $\| r ., r r$ & $1 \leqslant 0, r \mu$ & . ז'ו & $|r|, q v$ & $1 \% v, 1 \%$ & $\mid r_{0, \Lambda q}$ & $11 \leqslant, 4$ & 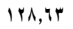 & $11 \Lambda, \varepsilon$ & $1 \cdot 1,17$ & يناير \\
\hline 10 & $1 \leqslant Y, Y 4$ & |r., & $1 \leqslant 0,41$ & r & IrY,rA & $1 \% v, 00$ & $M Y, Y V$ & $\ldots 110$ & $1 \mathrm{rq}, \cdot \mathrm{V}$ & $\| \Lambda, v \mid$ & $1 \cdot \Lambda, r \leqslant$ & فبر اير \\
\hline 10 & $1 \leqslant r, r_{0}$ & $1+., 01$ & $1 \leqslant 0, v_{0}$ & $1 r \leqslant, 9$ & IKY,\&Y & $|r v, r|$ & $\mid Y Y, Y A$ & $11 \leqslant, 94$ & $1 \times 9,19$ & $\| \Lambda, v \mid$ & I. A,YY & مارس \\
\hline $10 \leqslant, V \mid$ & $1 \leqslant r, q r$ & r|r & $1 \leqslant 4, r y$ & ץו, צזו & IrY,q0 & $1 \% \Lambda, 14$ & $1 Y 4, V 9$ & $110,\{r$ & Irq,V & $119,1 \leq$ & $1 \cdot 1,00$ & أبريل \\
\hline $10 \leqslant, 10$ & $1 \leqslant \mu, .0$ & or & $1 \leqslant 4,4$ & & Dr,.0 & $\mid r \Lambda, Y Y$ & $1 Y Y, \wedge 0$ & $110,\{4$ & Irq,q & $119, r \leq$ & $1 \cdot 1,01$ & مايو \\
\hline $10 \leqslant, 91$ & $1 \leqslant r, \mid v$ & $|r|, r v$ & $1 \leqslant 4,00$ & & SYr,I & $\| \psi_{1}, r_{0}$ & $1 \times 4, q$. & 110,44 & & $119, \mathrm{rV}$ & $1 \cdot 1, \leqslant 9$ & يونيو \\
\hline $10 \%, .1$ & Or, & \& & $1 \leqslant V, r y$ & Iro,q. & $1 Y \leqslant, 11$ & $\mid r q, \$ 1$ & $\mid r v, q r$ & 117,40 & $|r|, \cdot v$ & $M r_{., Y r}$ & $1.9,44$ & يوليه \\
\hline 101,09 & $1 \leqslant 4, \vee \wedge$ & דים & $10 ., 1 \leq$ & $\| \Psi \Lambda, \xi$. & IYY,TV & $1 \leqslant 1,9$ & $\mid r \cdot, r q q$ & $\| \Lambda, \wedge \Lambda$ & |rr,O & Irr, & $\| 11, v 1$ & أغسطس \\
\hline 109,71 & $1 \leqslant v, v q$ & $1 \times 0,91$ & 101,1 \& & $1 \% 9,\{$. & IrV,TY & $1 \leqslant 4, \wedge 9$ & o & 119,11 & $\mid r \leqslant, \Delta 1$ & $\mid r r, \Delta 1$ & $11 \mathrm{r}, \Delta \wedge$ & سبتمبر \\
\hline $17 ., 7 v$ & $1 \leqslant \wedge, \wedge$ ० & $\mid r v, \cdot r$ & 104,19 & צ & $1 \times 1,19$ & & Irr, & $M r, A$. & $1 r 0,0 q$ & Ir\&,Or & דוצ, & أكتوبر \\
\hline $171, \cdot r$ & $1 \leqslant 9,19$ & $\mid r v, r v$ & lor,or & $1 \leqslant, v y$ & $1 \times q, .$. & I & ITr,Y & $|r|, 0$ & $1 \times 0,99$ & $M \&, A V$ & $\| r, v_{0}$ & نوفبر \\
\hline $111, r 4$ & $1 \leqslant 9,0 \%$ & Irv,v. & $10 \%, \wedge r$ & $1 \leqslant 1,99$ & Irq,rr & $1 \leq\{$, Or & $|r r, q|$ & $|r|, r$. & • . & rro,rr & $11 \leqslant, 0$ & ليسمبر \\
\hline
\end{tabular}

المصدر : حسبت وجمعت من نتائج نماذج (ARIMA). 
بإعادة الكثف عن الارنباط الذاتي بين البو اقي في السلسلة الزمنية لأسعار لحوم جاموسـي العي

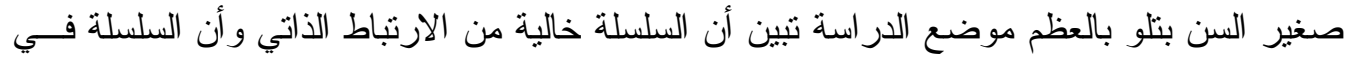

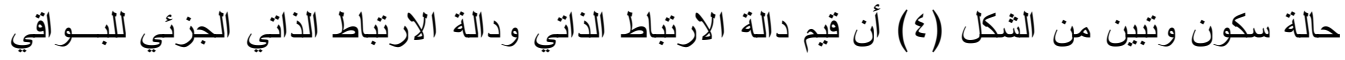
تقع جميعها بين الحد الأعلي و الحد الأدني بدرجة ثقة 90\% وتثنير نتائج التحليل إلي تحســن قيهــة اختبار Theil's U و التي قدرت بنحو 97، • بعد معالجة الارنباط الذاتي وسكون السلسلة الزمنية وهو

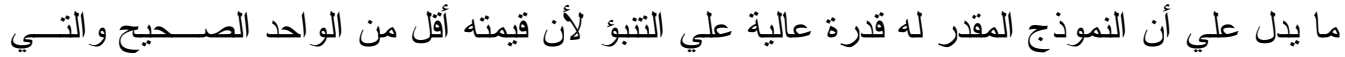
قدرت بنحو 0ء، قبل معالجة الارنباط الذاتي وعدم سكون السلسلة الزمنية.

شكل (ء) دالة الارتباط الذاتي ودالة الارتباط الذاتي الجزئي للبواقي لأسعار لحوم جاموسي صغير السن

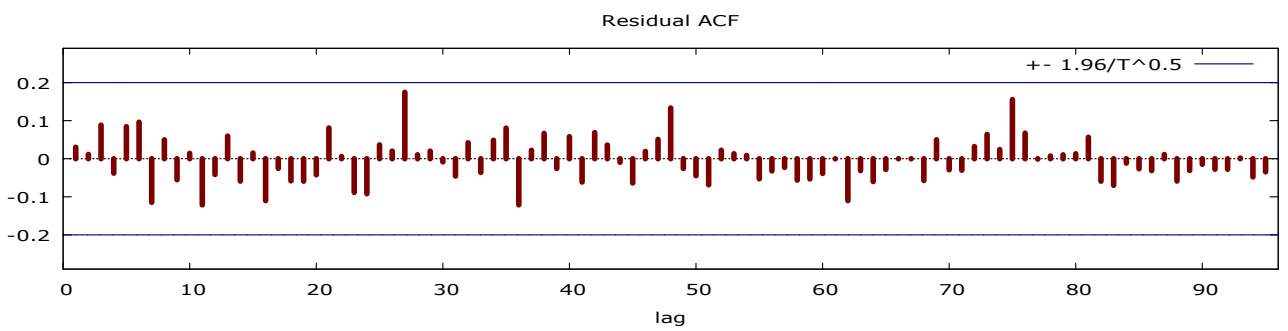

Residual PACF

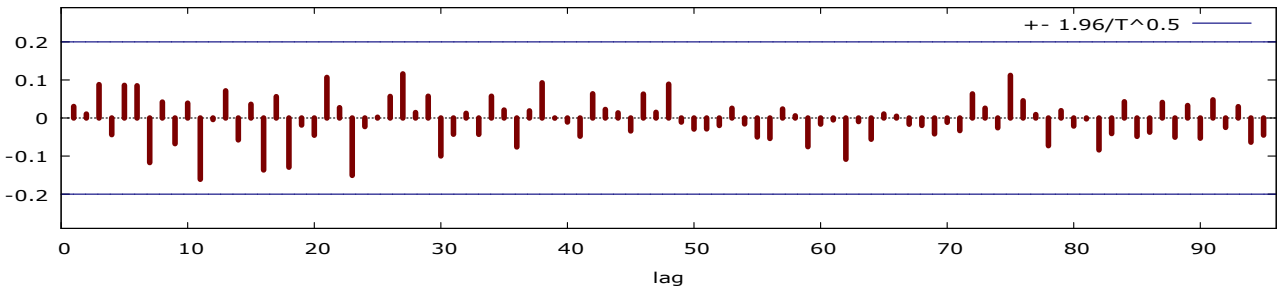

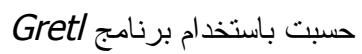

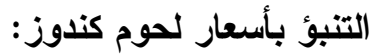

يوضح جدول (• ( ) معايير المفاضلة لنماذج (ARIMA) للتنبؤ بأسعار لحوم بقري وجاموسي منوسط السن (كندوز مشفي) أن نموذج (ARIMA) في المحاولة رقم (r) من أفضل النماذج وذللك لأن

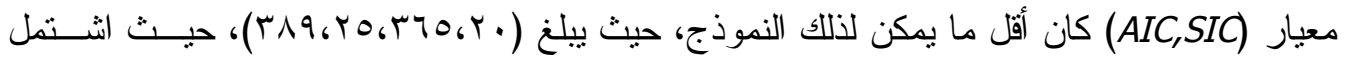

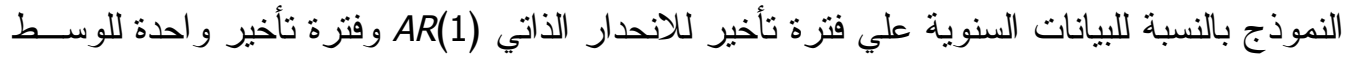

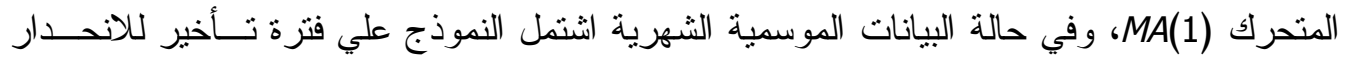
الذاتي الموسمي SAR(1) وفترة تأخير واحدة للوسط المتحرك الموسمي (SMA(1) و عمل فروق مسن

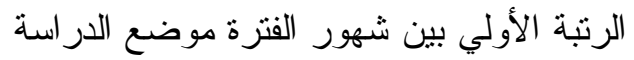


جدول ( • 1) معايير اختيار أفضل نماذج (ARIMA) للتنبؤ بأسعار لحوم كندوز

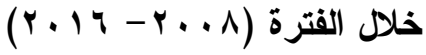

\begin{tabular}{|c|c|c|c|c|c|c|c|c|c|c|}
\hline \multirow{3}{*}{ Trying } & \multicolumn{9}{|c|}{ ARIMA Model } & \multicolumn{7}{c|}{ Model Selection } \\
\cline { 2 - 12 } & \multicolumn{3}{|c|}{ Non Seasonal } & \multicolumn{3}{c|}{ Seasonal } & \multicolumn{2}{c|}{} \\
\cline { 2 - 12 } & $P$ & $D$ & $Q$ & $P$ & $D$ & $Q$ & AIC & SIC & $L L$ & $Q H$ \\
\hline 1 & 0 & 1 & 2 & 1 & 0 & 3 & 365.86 & 389.93 & $173.93-$ & 375.62 \\
\hline 2 & 0 & 1 & 2 & 2 & 0 & 2 & 365.20 & 389.25 & $173.60-$ & 372.93 \\
\hline 3 & 0 & 1 & 2 & 2 & 0 & 3 & 366.95 & 393.68 & $173.48^{-}$ & 377.79 \\
\hline 4 & 0 & 1 & 2 & 2 & 0 & 4 & 368.81 & 398.21 & $173.41-$ & 380.73 \\
\hline
\end{tabular}

المصدر : حسبت وجمعت من نتائج نماذج (ARIMA).

يوضح جدول (I') نتائج تقدير المحاولة رقم (Y)، لنموذج (ARIMA) للتنبؤ بأسعار لحسوم

بقري وجاموسي متوسط السن (كندوز مشفي).

جدول(1 1 ) نموذج (ARIMA) للتبؤ بأسعار لحوم كندوز

\begin{tabular}{|c|c|c|}
\hline Model & Beta & Z-test \\
\hline Constant & 0.235 & $0.03^{-}$ \\
SAR1 & 0.347 & $0.91^{-}$ \\
SAR2 & $0.630-$ & $3.88^{-{ }^{*}}$ \\
MA1 & 0.248 & $2.43^{*}$ \\
MA2 & 0.101 & $1.06^{-}$ \\
SMA 1 & $0.463-$ & $1.18^{-}$ \\
SMA2 & 1 & $0.22^{-}$ \\
$I$ & 0.544 & $0.94^{-}$ \\
\hline
\end{tabular}

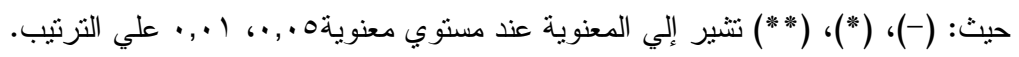

المصدر : حسبت وجمعت من ننائج نماذج (ARIMA).

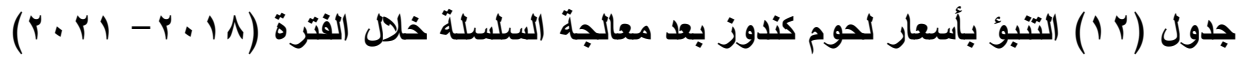

\begin{tabular}{|c|c|c|c|c|c|c|c|c|c|c|c|c|}
\hline \multicolumn{3}{|c|}{$r \cdot r)$} & \multicolumn{3}{|c|}{ r.r. } & \multicolumn{3}{|c|}{ r.19 } & \multicolumn{3}{|c|}{ r.1A } & \multirow[t]{2}{*}{ الشهور } \\
\hline حد أعلي & التنبؤ & حد أدني & حد أعلي & التنبؤ & حد أدني & حد أعلي & التنبؤ & حد أدني & حد أعلي & التنبؤ & حد أدني & \\
\hline $1 \wedge Y, \Lambda$ & $1 \bullet \Lambda, \Lambda$ & $1 \pi \leqslant, 1$ & $1 V_{\cdot}, 9$ & 101,1 & ITY,V & $10 \leqslant, 4$ & $1 \leqslant \cdot, Y$ & $1 Y q, \cdot$ & $1 \times 4,8$ & $1 Y 0,9$ & $110, r$ & بناير \\
\hline $1 \wedge \leqslant$, & $109, \mathrm{~V}$ & I & IVY,Y & lor, $V$ & Irr, & $100,\{$ & $1 \leqslant \cdot, \Lambda$ & $1 Y Y, r$ & $1 r v, 1$ & Iry, r & $110, r$ & فبر اير \\
\hline $\mid \Lambda_{0},\{$ & $14, \mathrm{~V}$ & $1 \times 4,1$ & IVT,V & lor,v & Irr,V & 10\%, & $1 \leqslant 1, r$ & $1 Y Y, r$ & Irv, & I & $110,\{$ & مارس \\
\hline $1 \wedge 4,0$ & 171,7 & $\mid r q, v$ & $\mid V \leqslant, V$ & $10 \leqslant, r$ & Irr, & lov,r & $1 \leqslant 1, \Lambda$ & $1 \times 4, r$ & $1 \times q, 1$ & Irv, & 118, & أبريل \\
\hline$|\wedge v|$, & $17 \%, \cdot$ & $1+4,1$ & $1 V_{0, \xi}$ & $10 \leqslant, 7$ & Irr,v & 109,0 & $1 \leqslant \%$, & $\mid r v, 1$ & $1 \leqslant 1, r$ & Irq,r & $11 v, 0$ & مايو \\
\hline $\mid \Lambda \Lambda$, & $17 \%, 0$ & Irv,. & $1 \vee 4,4$ & $100, r$ & צ & $19 ., 0$ & $1 \leqslant \leqslant, 1$ & $I r v, v$ & $1 \leqslant Y, 0$ & צ & $11 \Lambda, r$ & يونيو \\
\hline $1 \wedge q, r$ & ؛ & $1 r v, q$ & IVV,r & 100,1 & Irr,q & $171, r$ & $1 \leqslant \leqslant, 0$ & $I r v, v$ & $1 \leqslant \leqslant, 1$ & $|r|, v$ & $119, r$ & يوليه \\
\hline $19 ., 0$ & 174,9 & $1 \% v, q$ & $|V \Lambda|$, & $10 \%$, & Irr, & $14 \%, 4$ & $1 \leqslant 0, \xi$ & $\mid r \Lambda, r$ & $1 \leqslant 0,9$ & Irr, & $M r_{0}, 0$ & أغسطس \\
\hline 191,1 & $14 \leqslant, 1$ & $\mid r \wedge, \xi$ & $\mid \vee \wedge, \Lambda$ & $10 \%, r$ & Irr, & ؛ & $1 \leqslant 0,9$ & $\mid r \Lambda, r$ & $1 \leqslant V, 0$ & $\mid r \leqslant, 0$ & $|r|, 0$ & سبتمبر \\
\hline 194,1 & 170,1 & $1 \% q$, & $\mid \Lambda \cdot, r$ & lov, & O, & $17 \leqslant, V$ & $1 \leqslant 4, \wedge$ & $1 Y \Lambda, q$ & $1 \leqslant \Lambda, 1$ & A & $|r|, \gamma$ & أكتوبر \\
\hline 194,0 & 170,1 & $\mid r \wedge, \Lambda$ & $1 A \cdot, 9$ & $1 \leqslant V, 1$ & r & $14 \%, v$ & $1 \leqslant \Lambda, \xi$ & $1 \% \cdot, 1$ & 10,0 & Irv, & Irr,o & نو فمبر \\
\hline 194,0 & $170, \xi$ & $\mid r \Lambda, r$ & $|A|, \Lambda$ & $10 \wedge, 1$ & \& & $179, r$ & 10,4 & $|r|, 9$ & $10 Y, \Lambda$ & $1+4,$. & $1 r_{0, r}$ & ديسمبر \\
\hline
\end{tabular}

حسبت وجمعت من نتائج نماذج (ARIMA). 
بإعادة الكثثف عن الارتباط الذاتي بين البو اقي في السلسلة الزمنية لأســعار لحــوم بقــري

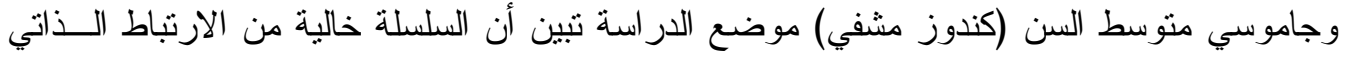

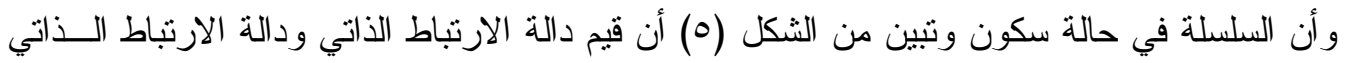
الجزئي للبو اقي تقع جميعها بين الحد الأعلي والحد الأدني بدرجة ثقة 90\% وتتشير نتائج التحليل إلي

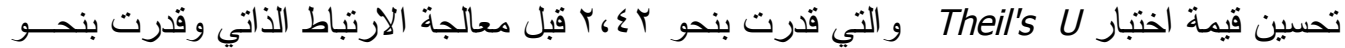
1^،، • بعد معالجة الارتباط الذاتي وسكون السلسلة الزمنية وهو ما يدل علي أن النموذج المقدر لــهـ قدرة عالية علي التنبؤ لأن قيمته اقل من الواحد الصحيح. شكل (0) دالة الارتباط الذاتي ودالة الارتباط الذاتي الجزئي للبو اقي لأسعار لحوم كندوز
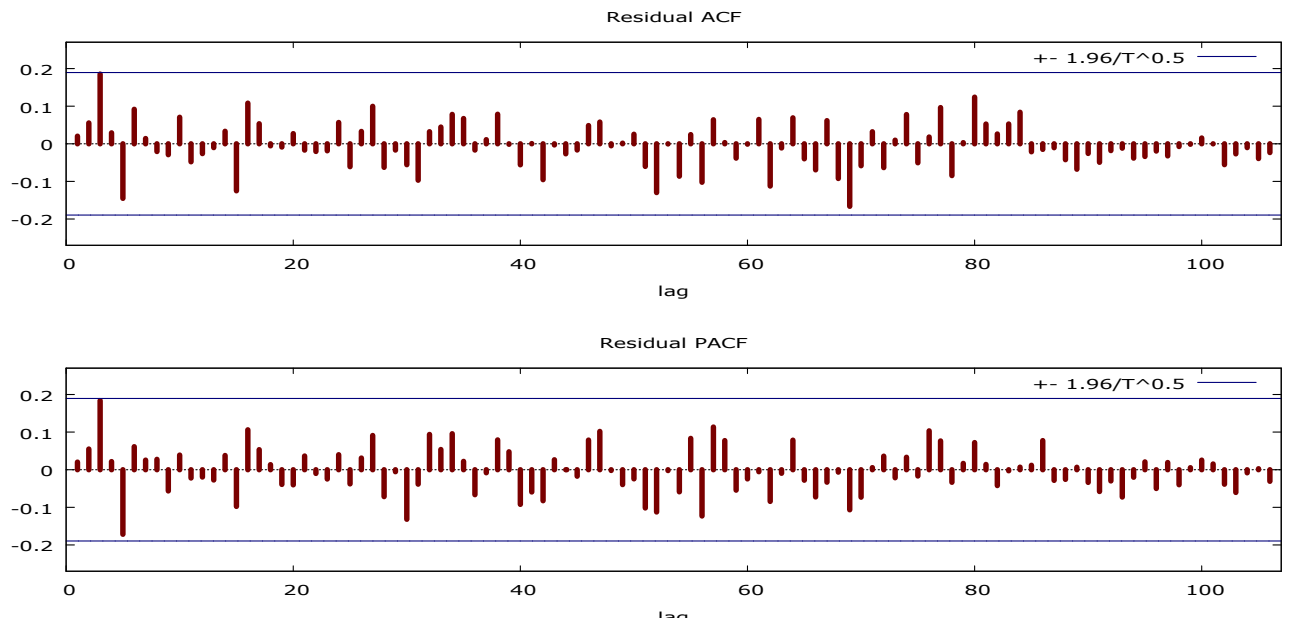

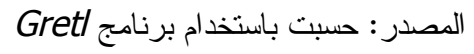

\section{التنبؤ بأسعار لحوم ضاني بلاي}

يوضح جدول (r ا) معايير المفاضلة لنماذج (ARIMA) للتنبؤ بأسعار لحوم ضــاني بلــدي

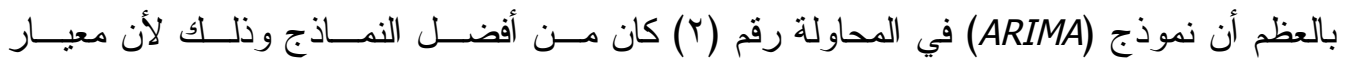
Sان اقل ما يمكن لذلك النموذج، حيث بلغ نحو (IIC,SIC) النموذج بالنسبة للبيانات السنوية علي فترتي تأخير للوسط المتحرك MA، وفي حالة البيانات الموسمية الثهرية انشتمل النموذج علي فترة تأخير للانحدار الذاتي الموسمي (1) SAR(1 وعل فروق من الرتبـــة

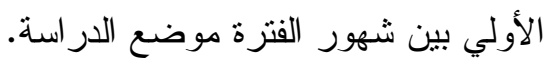

جلول (r I ) معايير اختيار أفضل نماذج (ARIMA) للتنبؤ بأسعار لحوم ضاني بلاي

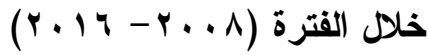

\begin{tabular}{|c|c|c|c|c|c|c|c|c|c|c|}
\hline \multirow[t]{3}{*}{ Trying } & \multicolumn{6}{|c|}{ ARIMA Model } & \multirow{2}{*}{\multicolumn{4}{|c|}{ Model Selection }} \\
\hline & & Seas & & & $75 O$ & & & & & \\
\hline & $P$ & $D$ & $Q$ & $P$ & $D$ & $Q$ & $\overline{A I C}$ & SIC & LL & $Q H$ \\
\hline 1 & 0 & 1 & $\frac{v^{2}}{2}$ & 1 & 0 & $\begin{array}{l}e^{\prime} \\
1\end{array}$ & 368.53 & 387.24 & $177.26-$ & 376.11 \\
\hline 2 & 0 & 1 & 2 & 1 & 0 & 0 & 366.82 & 382.85 & 177.41- & 373.32 \\
\hline 3 & 0 & 1 & 3 & 1 & 0 & 0 & 368.18 & 386.89 & 177.09- & 375.76 \\
\hline 4 & 0 & 1 & 1 & 1 & 0 & 1 & 367.21 & 380.58 & $178.61-$ & 372.62 \\
\hline 5 & 0 & 0 & 1 & 1 & 0 & 0 & 479.68 & 493.09 & $234.84-$ & 485.12 \\
\hline
\end{tabular}

حسبت وجمعت من نتائج نماذج (ARIMA). 
يوضح جدول (ع ا) نتائج تقدير المحاولة رقم (Y)، نموذج (ARIMA) للتنبؤ بأسعار لحـوم

ضاني بلدي.

جدول( 1 ) ) نموذج (ARIMA) للتنبؤ بأسعار لحوم ضاني بلدي

\begin{tabular}{|c|c|c|}
\hline Model & Beta & Z-test \\
\hline Constant & 0.17 & $0.98^{-}$ \\
SAR1 & 0.08 & $0.47^{-}$ \\
MA1 & 0.12 & $0.24^{-}$ \\
MA2 & 0.18 & $0.07^{*}$ \\
$I$ & 0.60 & $0.94 \varepsilon^{-}$ \\
\hline
\end{tabular}

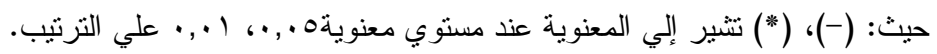

حسبت وجمعت من نتائج نماذج (ARIMA).

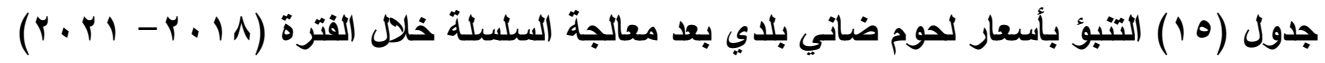

\begin{tabular}{|c|c|c|c|c|c|c|c|c|c|c|c|c|}
\hline \multicolumn{3}{|c|}{$r, r)$} & \multicolumn{3}{|c|}{ T.Y. } & \multicolumn{3}{|c|}{5.19} & \multicolumn{3}{|c|}{$r .11$} & \multirow[t]{2}{*}{ الشهور } \\
\hline حد أعلي & التنبؤر & حد أدني & حد أعلي & التنبؤ & حد أدني & حد أعلي & التنبؤُ & حد أدني & حد أعلي & التنبئز & حد أدني & \\
\hline $1 \vee 9, .0$ & 100,11 & $1+1,11$ & $174,0$. & $1 \leqslant 0, \wedge q$ & $\mid r 0, Y \Lambda$ & lor, ra & $1 \times 4,74$ & $T r_{\mu},+r$ & $|r \wedge, V|$ & lrv,r. & $110, \Lambda \Lambda$ & يناير \\
\hline $\mid A \cdot, \cdot V$ & $100, \mathrm{AN}$ & 1r1, & $17 \mathrm{~V}, 04$ & $1 \leq 4,74$ & ryo, vo & $10 \leqslant, \leqslant$ & Irv, & $\mid r \cdot, \leqslant \psi$ & $1 \leqslant ., 0$ & IrA, Ir & 119,19 & فبر لير \\
\hline$|A|, 1$. & 107,70 & $|r Y, Y|$ & $M$ & $1 \leqslant V, \leqslant \psi$ & Iry,Yr & 100,00 & $\| r \Lambda, r$. & $\mid r \cdot, \Lambda \leqslant$ & $1 \leqslant 1, r r$ & $\mid r \wedge, \wedge q$ & $114, \$ V$ & مارس \\
\hline IAY,IY & $10 \mathrm{~V}, \leqslant \mathrm{r}$ & IrY,VY & 179,71 & $1 \leqslant \Lambda, r$. & $|r q, v|$ & 104,4 & $\mid r \wedge, q \vee$ & $|r|, Y \mid$ & $1 \leqslant r, O V$ & $1 \times 9,14$ & $114, v_{0}$ & أبريل \\
\hline IAr,Ir & 101,19 & צ & $V V_{\cdot, V}, \mathrm{~s}$ & $1 \leqslant \Lambda, 94$ & $1 Y v, 19$ & $10 \mathrm{~V}, \mathrm{Vq}$ & Irq,Vr & $|r|, 4$ & $|\leqslant r, \Lambda|$ & Ir., & $118, .4$ & مايو \\
\hline $1 A \leqslant, 10$ & 101,97 & TrT, & $|v|, v q$ & $1 \leqslant q, V \mu$ & $\mid \gamma v, \eta$ & $10 \wedge, \wedge 9$ & $1 \leqslant, 0$. & $|Y Y, 1|$ & $1 \leqslant 0, \cdot r$ & $|r|, r$. & $\| \mathrm{V}, \mathrm{rA}$ & يونيو \\
\hline $11_{0,11}$ & $109, \mathrm{Vr}$ & $1 r \leqslant, r q$ & IVY,AF & $10 ., 0$. & $|Y \Lambda| V$, & 17. & $1 \leqslant 1, r v$ & IrY,O0 & $1 \leqslant Y, r r$ & $1 \times 1,94$ & $\|v, v\|$ & يوليه \\
\hline $1 A 4,1 V$ & $19 ., 49$ & $\mid r \&, A r$ & $\mid V \Psi, A \Lambda$ & $101, Y \mathrm{~V}$ & $\mid Y \Lambda, Y Y$ & $171, .9$ & $1 \leqslant r,+\leqslant$ & $1 Y r, q 9$ & $1 \leqslant V, \leqslant 1$ & IrY,Vr & $111, \cdot 0$ & أغسطس \\
\hline IAV,IA & $171, \mathrm{r}$ & oro,ro & $\mid V \leqslant, q Y$ & $10 \%, ., 5$ & $1 \times 9,19$ & $17 r, 11$ & $|\leqslant r, A|$ & \& & $1 \leqslant \Lambda, 71$ & Irr,or & $11 \wedge, \llbracket ؛$ & سبتمبر \\
\hline $1 A \Lambda, 19$ & $17 r, . r$ & $1 r_{0, \Lambda \Lambda}$ & $1 v_{0,94}$ & $10 \%, A 1$ & $1 \times 9,79$ & Vץr & $1 \leqslant r, \otimes 1$ & IrY,Aq & $1 \leqslant 9, \vee \wedge$ & $1 r \leqslant, r 9$ & $\| \wedge, \wedge$ & أكتوبر \\
\hline 119,19 & $17 r, \Lambda$. & $\mid r 4,\{\mid$ & $1 \vee 4,99$ & $10 \%, 01$ & $17 ., 17$ & D & $1 \leqslant \leqslant, r_{0}$ & Irs, & 10,97 & $1 r_{0,1}$ & $119, r 1$ & نوفمبر \\
\hline $19 ., 19$ & $17 r, o v$ & $1 \times 4,90$ & $I V \Lambda,+Y$ & צ & Ir., TV & $190, \leqslant 4$ & $1 \leqslant 0,1 Y$ & $M \&, A r$ & lor,1 & $1 r_{0, \Lambda \Lambda}$ & 119,74 & لبيسمبر \\
\hline
\end{tabular}

المصدر : حسبت وجمعت من نتائج نماذج (ARIMA).

بإعادة الكثف عن الارتباط الذاتي بين البو اقي في السلسلة الزمنية لأسعار لحــوم ضــاني

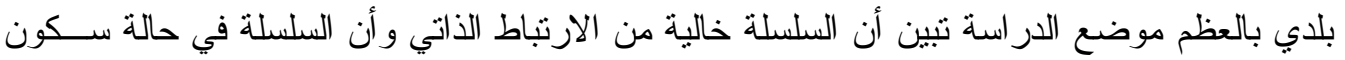
وتبين من الثكل (T) أن قيم دالة الارتباط الذاتي ودالة الارتباط الذاتي الجزئي للبو اقي تقع جميعهـا بين الحد الأعلي و الحد الأدني بدرجة ثقة 90\% وتنتير نتائج التحليل إلي تحســين قيمــة اختبــار

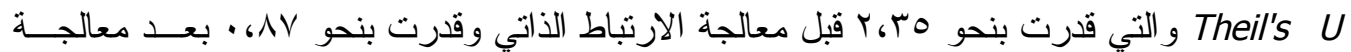

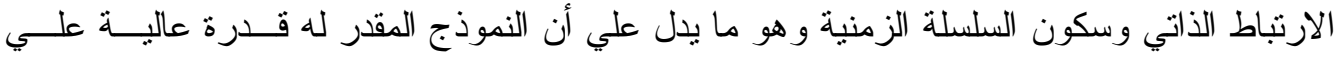
النتبؤ لأن قيمته أقل من الو احد الصحيح. 
شكل (†) دالة الارتباط الذاتي ودالة الارتباط الذاتي الجزئي للبو اقي لأسعار لحوم ضاني بلاي بالعظم

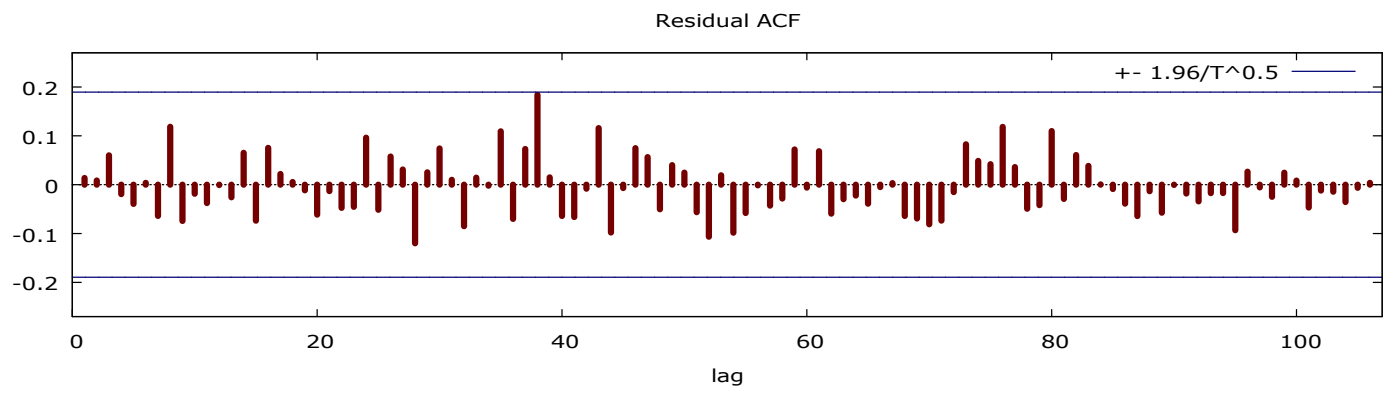

Residual PACF

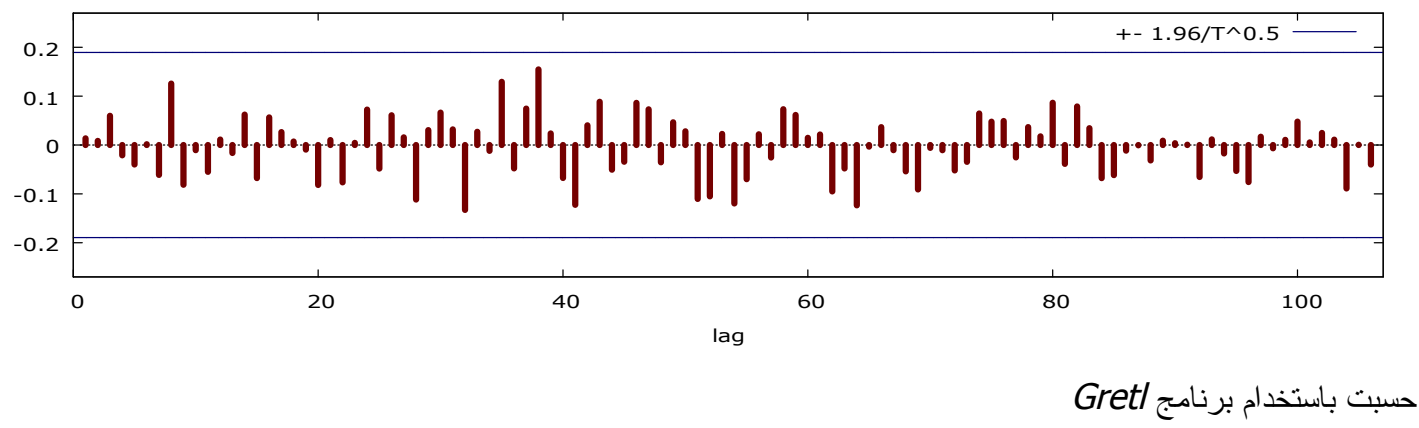

توصية

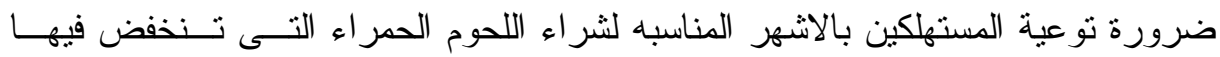
الاسعار وفقا للعرض و الطلب.

المراجع

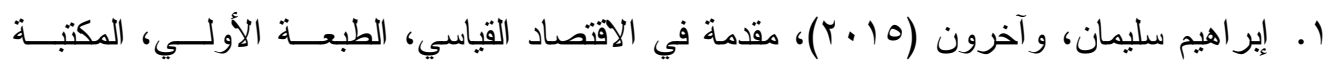
الأكاديمية.

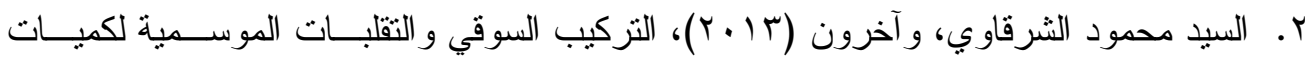

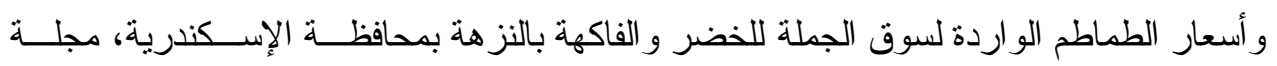
الإسكندرية للبحوث الزر اعية، المجلد (ب^ه)، العدد(ب).

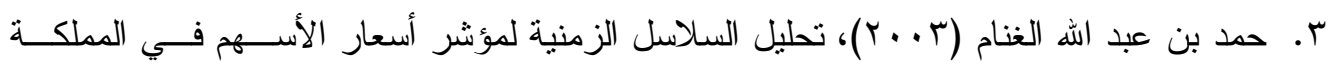

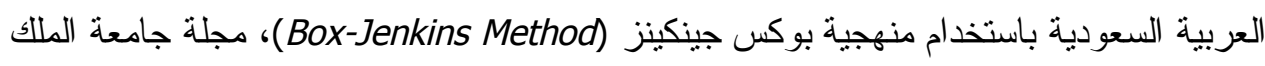

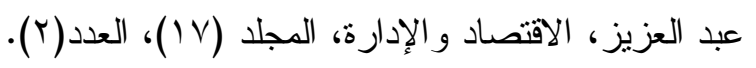

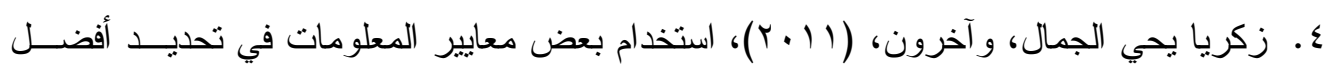

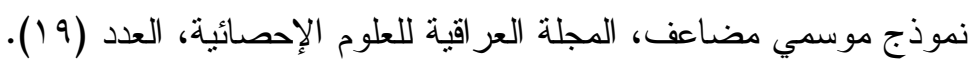

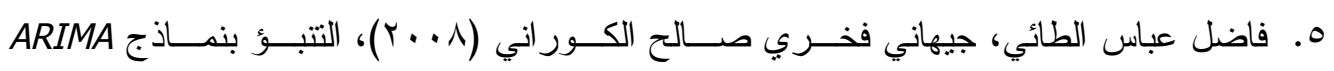

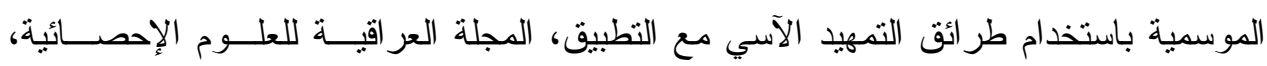
العدد (ع ا ). 7. . والترفاندل (ب99 ())، السلاسل الزمنية من الوجهة التطبيقية ونماذج بوكس -جنكنز، دار المريخ للنشر ، الرياض المملكة العربية السعودية. 


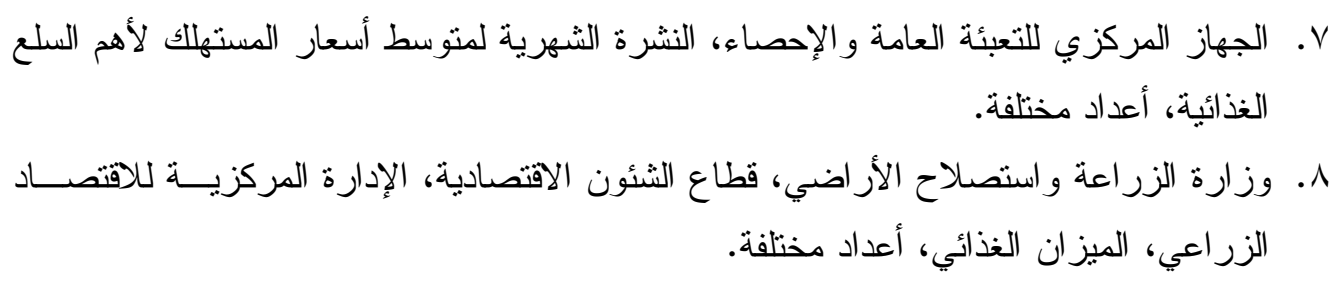

9. Box, G. and Jenkins, G., (1976) "Time Series Analysis: Forecasting and Control", San Francisco. Calif, Holden- Day,U.S.A.

10. Box, G. E. and Price, D. A. (1970), " Distribution of Residual Autocorrelations in Autoregressive - Integrated Moving Average Time Series Models " , JASA, Vol.55, No.332, PP.1509-1525.

11. Blieme .F,(1973) "Theils Forecast Accuracy Coefficient: A Clarification" Journal of Marketing Research,Vol.X (November), 444-446.

12. Brock Well , P.J. and Davis, R.A. (1991), " Time Series Theory and Methods " ,2nd ed, Springer Verlag New York Inc, New York. 
